\title{
Scalar torsion and a new symmetry of general relativity
}

\author{
J. B. Fonseca-Neto, ${ }^{*}$ C. Romero ${ }^{\dagger}$ and S. P.G. Martinez ${ }^{\ddagger}$
}

November 3, 2018

\begin{abstract}
We reformulate the general theory of relativity in the language of RiemannCartan geometry. We start from the assumption that the space-time can be described as a non-Riemannian manifold, which, in addition to the metric field, is endowed with torsion. In this new framework, the gravitational field is represented not only by the metric, but also by the torsion, which is completely determined by a geometric scalar field. We show that in this formulation general relativity has a new kind of invariance, whose invariance group consists of a set of conformal and gauge transformations, called Cartan transformations. These involve both the metric tensor and the torsion vector field, and are similar to the well known Weyl gauge transformations. By making use of the concept of Cartan gauges, we show that, under Cartan transformations, the new formalism leads to different pictures of the same gravitational phenomena. We show that in an arbitrary Cartan gauge general relativity has the form of a scalar-tensor theory. In this approach, the RiemannCartan geometry appears as the natural geometrical setting of the general relativity theory when the latter is viewed in an arbitrary Cartan gauge. We illustrate this fact by looking at the one of the classical tests of general relativity theory, namely the gravitational spectral shift. Finally, we extend the concept of space-time symmetry to the more general case of Riemann-Cartan space-times endowed with scalar torsion. As an example, we obtain the conservation laws for auto-parallel motions in a static spherically symmetric vacuum space-time in a Cartan gauge, whose orbits are identical to Schwarzschild orbits in general relativity. PACS numbers: 04.20.-q, 04.20.Cv, 04.50.Kd
\end{abstract}

keywords: torsion; general relativity; conformal transformations.

address: Departamento de Física, Universidade Federal da Paraíba, João Pessoa, PB 58059-970, Brazil

\footnotetext{
*jfonseca@fisica.ufpb.br

${ }^{\dagger}$ cromero@fisica.ufpb.br

${ }^{\ddagger}$ sgomezm@fisica.ufpb.br
} 


\section{Introduction}

As we know, Einstein's general relativity is a geometric theory of gravitation in which the gravitational field appears as a manifestation of the space-time curvature. According to general relativity, space-time is represented by a $(3+1)$-dimensional differentiable manifold endowed with a Lorentzian metric and a Levi-Civita connection, the latter being completely determined by the former. However, it is possible to consider a more general geometrical setting, in which the metric and the connection may be taken as independent structures. A general metric-compatible connection has two important properties: curvature and torsion [1, 2]. Torsion is a priori absent in the geometrical framework of general relativity, although it was taken into consideration by Einstein in his search for a unified theory of gravitation and electromagnetism [3]. Since then, the meaning and role of torsion in gravitation has been a recurring theme. Initially, Cartan suggested that torsion could be related to the intrinsic spin of elementary particles. This idea has developed into the Einstein-Cartan theory, where not only mass, but also the intrinsic spin of matter were considered as source of the gravitational field [4]. Further generalization led to Poincarï $\_\frac{1}{2}$ gauge theories [1], in which Lagrangians with quadratic terms in the curvature and in the torsion have been considered. In all these theories, torsion is regarded as an independent geometrical structure, which is related to the intrinsic spin of elementary particles and expected to predict new gravitational effects. In connection with this, a teleparallel theory of gravitation was developed, where the presence of the gravitational field appears as a manifestation of the torsion in a space-time with no Riemann-Cartan curvature. This approach is now known as the teleparallel equivalent of general relativity (TEGR), where only mass is assumed to be the source of torsion, which, in turn, is no longer related to the intrinsic spin of elementary particles [5]. Finally, let us mention the recent $\mathrm{f}(\mathrm{T})$ theories of gravitation [5], which are recent generalizations of TEGR analogous to the recent $f(R)$ theories, mainly developed to address open questions, such as, the origin of dark matter and dark energy [6]. These efforts have given rise to the so-called extended theories of gravity [7] .

In this work, we are concerned with the meaning and role of torsion in gravity theories from a different point of view by considering two questions of a rather general character. The first question is: Is it possible to enlarge the symmetries of general relativity in order to include conformal transformations of the metric of space-time? The answer to this question seems to have some relevance as it has been argued recently that a conformal theory of gravity would be a viable candidate for a quantum theory of gravity, since it is expected to be renormalizable [8]. The second questions is: Is it possible to formulate general relativity in the framework of a Riemann-Cartan space-time? This is related to the more general question: To what extent is Riemannian geometry the only possible geometrical setting for general relativity? In this paper, we will try to address, at least partially, these two questions.

As is well known, Einstein's theory of gravity in its original formulation is not invariant under conformal transformations. One reason for this is that the geometrical language of Einstein's theory is completely based on Riemannian geometry. Indeed, for a long time general relativity has been inextricably associated with the geometry of Riemann. Further developments, however, have led to the discovery of different geometrical structures, which 
we might generically call "non-Riemannian" geometries. Many of these developments were closely related to attempts at unifying gravity with electromagnetism [3]. Our main objective in this paper is to show that one can formulate general relativity using the language of a non-Riemannian geometry, namely, the Riemann-Cartan geometry with a vectorial torsion. In this formulation, general relativity appears as a theory in which the gravitational field is described simultaneously by two geometrical fields: the metric tensor and the torsion field, the latter being an essential part of the geometry, manifesting its presence in almost all geometrical phenomena, such as curvature, geodesic motion, and so on. As we will see, in this new geometrical setting general relativity exhibits a new kind of invariance, namely, the invariance under Cartan transformations.

The outline of this paper is as follows. We begin by presenting, in Section 2, the basic mathematical facts of Riemann-Cartan geometry and the concept of Cartan gauge. In Section 3, we show how to formulate general relativity in a Riemann-Cartan spacetime endowed with scalar torsion. In this formulation, we will see that the theory is invariant under the group of Cartan transformations. We devote Section 4 to examine the Newtonian limit, which will help us to get some insight into the role of the scalar torsion field in the Cartan representation of general relativity. Then, in Section 5 we illustrate how different pictures of the same phenomena may arise in distinct gauges by considering the description of the gravitational spectral shift in a Cartan gauge. In Section 6 , we extend the concept of space-time symmetry to the more general case of RiemannCartan space-times with scalar torsion. As an example, we obtain the conservation laws for auto-parallel motion and determine the orbits of test particles, in a static spherically symmetric vacuum space-time in a Cartan gauge, in the equivalence class of Schwarzschild space-time. Section 7 contains our final remarks.

\section{Riemann-Cartan space-time and scalar torsion}

In this section, we briefly review some basic properties of Riemann-Cartan space-times. We will also introduce the notations and conventions that will be used in this paper. As is well known, in a general affine geometry the metric and the connection are independent structures. The metric defines the concepts of length and angle between two vectors, while the connection leads to the concept of parallel transport and covariant derivative. In such a geometry, three tensors are of special interest. The first two are the torsion tensor and the curvature tensor, both depending only on the connection. The components of the torsion tensor are given, in a coordinate basis, by the antisymmetric part of the connection coefficients $\mathcal{T}_{\mu \nu}^{\alpha}=\Gamma_{\mu \nu}^{\alpha}-\Gamma_{\nu \mu}^{\alpha}$. On the other hand, the components of the curvature tensor are given by $R_{\beta \mu \nu}^{\alpha}=\Gamma_{\mu \beta, \nu}^{\alpha}-\Gamma_{\nu \beta, \mu}^{\alpha}+\Gamma_{\nu \rho}^{\alpha} \Gamma_{\mu \beta}^{\rho}-\Gamma_{\mu \rho}^{\alpha} \Gamma_{\nu \beta}^{\rho}$. The covariant derivative of a contravariant vector field $u^{\mu}$, in a coordinate basis, will be given by $\nabla_{\alpha} u^{\mu}=u_{; \alpha}^{\mu}=$ $u_{, \alpha}^{\mu}+\Gamma_{\alpha \nu}^{\mu} u^{\nu}$, where $u_{, \alpha}^{\mu}$ denotes partial derivatives. In the presence of curvature and torsion, the second covariant derivatives do not commute and satisfy the Ricci identities $\left(\nabla_{\beta} \nabla_{\alpha}-\nabla_{\alpha} \nabla_{\beta}\right) u^{\mu}=R_{\lambda \alpha \beta}^{\mu} u^{\lambda}+\mathcal{T}_{\alpha \beta}^{\lambda} \nabla_{\lambda} u^{\mu}$ and $\left(\nabla_{\beta} \nabla_{\alpha}-\nabla_{\alpha} \nabla_{\beta}\right) f=\mathcal{T}^{\lambda}{ }_{\alpha \beta} \nabla_{\lambda} f$, where $f$ is a scalar field. Finally, the third important tensor is the non-metricity tensor defined by the covariant derivative of the metric $Q_{\alpha \mu \nu}=\nabla_{\alpha} g_{\mu \nu}$, which depends on both the metric and the connection (see, for instance, [1, 2] and references therein). 
Let us recall that the space-time of general relativity corresponds to the special case in which both the torsion and non-metricity tensors vanish. The first condition implies that, in a coordinate basis, the connection is symmetric, while the second condition assures that the connection is compatible with the metric, that is, lengths and angles between vectors are assumed to be preserved under parallel transport. We are thus led to the LeviCivita connection $\widetilde{\Gamma}$, whose components are known as the Christoffel symbols of second kind and are given by $\widetilde{\Gamma}_{\mu \nu}^{\alpha}=\left\{\begin{array}{c}\alpha \\ \mu \nu\end{array}\right\}=\frac{1}{2} g^{\alpha \beta}\left(g_{\beta \nu, \mu}+g_{\mu \beta, \nu}-g_{\mu \nu, \beta}\right) 1$. We, thus, see that in the Riemannian space-time of general relativity the curvature tensors and all geometric properties of the space-time constructed with $\widetilde{\Gamma}$ will depend only on the metric.

It turned out that shortly after the formulation of general relativity, the desire to develop a unified and geometrized theory of gravitation and electromagnetism led physicists to weaken the two restrictive conditions mentioned above. This would allow to increase the number of degrees of freedom coming exclusively from geometry. In this way, the first non-Riemannian manifolds were developed (for details see [3] and references therein). Let us mention two of them. The first is Weyl geometry, where torsion is absent, while nonmetricity is present. In this case, the non-metricity tensor is given by $Q_{\alpha \mu \nu}=\omega_{\alpha} g_{\mu \nu}$, where $\omega_{\alpha}$ is a covariant vector defined on the manifold [9]. Weyl identified the vector $\omega_{\alpha}$ with the electromagnetic potential, while the usual gauge transformations of electromagnetism appear in a natural way as part of the so-called Weyl transformations. Although his unified theory is no longer considered viable from the standpoint of physics, Weyl geometry has been revived in a particular version, known as the Weyl integrable geometry, when $\omega_{\alpha}$ corresponds to the gradient of a scalar field [10]. Weyl integrable geometry has received a lot of attention in the recent years as the mathematical framework of some alternative gravity theories [11]. The second example of a non-Riemannian geometrical structure is due to H. Cartan and is called Riemann-Cartan geometry [4]. Here, the space-time manifold is allowed to possess torsion, while the connection is required to be compatible with the metric, i.e. $Q_{\alpha \mu \nu}=0$, the connection has only one restriction: it must be compatible with the metric. In this case, the metric compatibility condition allows the Riemann-Cartan connection to be written as $\Gamma_{\mu \nu}^{\alpha}=\left\{\begin{array}{c}\alpha \\ \mu \nu\end{array}\right\}+K_{\mu \nu}^{\alpha}$, where $K_{\mu \nu}^{\alpha}=\frac{1}{2} g^{\alpha \beta}\left(g_{\rho \mu} \mathcal{T}_{\beta \nu}^{\rho}+g_{\rho \nu} \mathcal{T}_{\beta \mu}^{\rho}+g_{\rho \beta} \mathcal{T}_{\mu \nu}^{\rho}\right)$ is the contortion tensor. Let us remark at this point that in a Riemann-Cartan manifold there are two equivalent sets of independent fundamental geometric objects, namely, $\left\{g_{\mu \nu}, \Gamma_{\mu \nu}^{\alpha}\right\}$ and $\left\{g_{\mu \nu}, T_{\mu \nu}^{\alpha}\right\}$, since the connection is completely determined by both the metric and the torsion.

An interesting fact is that, in a Riemann-Cartan space-time, there are transformations involving both the metric and the connection, which preserve the metric compatibility condition. In addition, these transformations leave invariant the curvature and, at the same time, change the torsion in a way similar to a gauge transformation [12. They are defined by the combined effect of a conformal transformation of the metric

$$
\bar{g}_{\mu \nu}=e^{f} g_{\mu \nu}
$$

where $f$ is a function of the coordinates, and the so-called Einstein's $\lambda$-transformations

\footnotetext{
${ }^{1}$ From now on, we use tilde superscripts to denote any quantity that depends only on the metric $g_{\mu \nu}$ and/or the Christoffel symbols calculated with $g_{\mu \nu}$. The metric assignature is $(+---)$.
} 
of the components of the connection [13]

$$
\bar{\Gamma}_{\mu \nu}^{\alpha}=\Gamma_{\mu \nu}^{\alpha}+\frac{1}{2} f_{, \mu} \delta_{\nu}^{\alpha}
$$

Note that (11) and (2) do not involve coordinate transformations. It is easy to verify that under these transformations, the components of the torsion transform according to

$$
\overline{\mathcal{T}}_{\mu \nu}^{\alpha}=\mathcal{T}_{\mu \nu}^{\alpha}-\frac{1}{2}\left(\delta^{\alpha}{ }_{\mu} f_{, \nu}-\delta^{\alpha}{ }_{\nu} f_{, \mu}\right)
$$

Conversely, under both the conformal transformation of the metric (11) and the transformation of torsion (3) the connection transforms according to an Einstein's $\lambda$-transformations (2). Henceforth we will refer to (11) of $g_{\mu \nu}$ and (3) as Cartan transformations.

Clearly, the components of curvature tensor of the Riemann-Cartan space-time are left invariant under Cartan transformations, that is, $\bar{R}_{\beta \mu \nu}^{\alpha}=R_{\beta \mu \nu}^{\alpha}$. On the other hand, it is evident that the Ricci tensor is also invariant, i.e. $\bar{R}_{\beta \nu}=\bar{R}_{\beta \lambda \nu}^{\lambda}=R_{\beta \nu}$, while the curvature scalar transforms as $\bar{R}=\bar{g}^{\mu \nu} \bar{R}_{\mu \nu}=e^{-f} R$. Finally, it follows from the previous transformations that the Weyl tensor $W_{\beta \mu \nu}^{\alpha}=R_{\beta \mu \nu}^{\alpha}-\frac{1}{2}\left(\delta_{\mu}^{\alpha} R_{\beta \nu}-\delta_{\nu}^{\alpha} R_{\beta \mu}-g_{\beta \mu} R_{\nu}^{\alpha}+\right.$ $\left.g_{\beta \nu} R_{\mu}^{\alpha}\right)-\frac{1}{6}\left(\delta_{\mu}^{\alpha} g_{\beta \nu}-\delta_{\nu}^{\alpha} g_{\beta \mu}\right) R$ is also left invariant.

As is known, the torsion tensor can be decomposed into three independent irreducible parts: the tensorial, the vectorial and the axial-vectorial parts [1, 14. Here, we will consider the decomposition into only two parts, namely, the vectorial torsion, which is determined by the torsion trace defined by $\mathcal{T}_{\nu}=\mathcal{T}^{\alpha}{ }_{\alpha \nu}$, and the traceless part $L^{\alpha}{ }_{\mu \nu}$ (including both the tensorial and axi-vetorial parts). In this way, we have

$$
\mathcal{T}_{\mu \nu}^{\alpha}=L_{\mu \nu}^{\alpha}+\frac{1}{3}\left(\delta_{\mu}^{\alpha} \mathcal{T}_{\nu}-\delta^{\alpha}{ }_{\nu} \mathcal{T}_{\mu}\right)
$$

Using the above decomposition we can verify that, under a transformation given by (1) and (2), the trace of the torsion effectively plays the role of a gauge vector field, whereas the traceless part is left invariant, that is,

$$
\bar{L}_{\mu \nu}^{\alpha}=L_{\mu \nu}^{\alpha}, \quad \overline{\mathcal{T}}_{\mu}=\mathcal{T}_{\mu}-\frac{3}{2} f_{, \mu} .
$$

Let us now assume that the Riemann-Cartan space-time we are considering is characterized by the following conditions: First, we require that the traceless part of the torsion vanishes identically $L_{\mu \nu}^{\alpha}=0$. Second, let us suppose the existence of a scalar field $\phi(x)$, which completely determines $\mathcal{T}_{\mu}$ through the equation

$$
\mathcal{T}_{\mu}=-\frac{3}{2} \phi_{, \mu}
$$

that is, the torsion trace $\mathcal{T}_{\mu}$ is the gradient of a scalar "potential" $\phi(x)$. This torsion determined by the gradient of a propagating potential, also called gradient torsion [15], will be referred to as scalar torsion, while the scalar field $\phi(x)$ will be called torsion scalar field. Thus, the Riemann-Cartan space-time with scalar torsion is characterized by two 
fundamental geometric objects, namely, the metric $g_{\mu \nu}$ and the torsion scalar field $\phi$, which transforms under (11) and (3) as

$$
\bar{g}_{\mu \nu}=e^{f} g_{\mu \nu}, \bar{\phi}=\phi+f .
$$

Since the transformations (77) are mappings between Riemann-Cartan space-times with scalar torsion, they will called Cartan (gauge) transformations. The set $(M, g, \phi)$ consisting of a differentiable space-time manifold $M$ endowed with a Lorentzian metric $g$ and a torsion scalar field $\phi$ will be referred to as a Cartan gauge. Any Cartan gauge $(M, g, \phi)$ is related to another $(\bar{M}, \bar{g}, \bar{\phi})$ by a Cartan transformation. It is easy to see that by means of a Cartan transformation (7) it is possible to go from an arbitrary Cartan gauge $(M, g, \phi)$ to a unique Cartan gauge $(M, \widehat{g}, \widehat{\phi})=(M, \widehat{g}, 0)$, where $\widehat{\phi}=0$, the latter being called Riemann gauge, since in this gauge the torsion vanishes. 2

As we will see in the next section, the Riemann gauge plays a fundamental role in the formulation of general relativity in an arbitrary Riemann-Cartan space-time with a scalar torsion. In fact, we will take advantage of a very important property of the Riemann gauge, which will be used as a heuristic tool to formulate general relativity in an arbitrary Riemann-Cartan space-time with a scalar torsion. Consider a Cartan gauge $(M, g, \phi)$ and the unique Riemann gauge $(M, \widehat{g}, 0)$, which is related to it by a Cartan transformation. It is not difficult to verify that the metric $\widehat{g}_{\mu \nu}$ and the connection $\widehat{\Gamma}_{\mu \nu}^{\alpha}=$ $\left\{\begin{array}{c}\alpha \\ \mu \nu\end{array}\right\}_{\widehat{g}}=\frac{1}{2} \widehat{g}^{\alpha \beta}\left(\widehat{g}_{\beta \nu, \mu}+\widehat{g}_{\mu \beta, \nu}-\widehat{g}_{\mu \nu, \beta}\right)$ of the Riemann gauge $(M, \widehat{g}, 0)$ can be expressed in terms of the metric $g_{\mu \nu}$ and the connection $\Gamma_{\mu \nu}^{\alpha}$ of the Cartan gauge $(M, g, \phi)$, respectively, through the relations

$$
\widehat{g}_{\mu \nu}=e^{-\phi} g_{\mu \nu}\left(\widehat{g}^{\alpha \beta}=e^{\phi} g^{\alpha \beta}\right), \widehat{\Gamma}_{\mu \nu}^{\alpha}=\Gamma_{\mu \nu}^{\alpha}-\frac{1}{2} \phi_{, \mu} \delta_{\nu}^{\alpha},
$$

which are invariant with respect to Cartan transformations. Therefore, these relations allow one to express quantities calculated in the Riemann gauge in terms of quantities in the Cartan gauge, without any reference to the Riemann gauge. This is an important property of the Riemann gauge, since not only $\widehat{g}_{\mu \nu}$ and $\widehat{\Gamma}_{\mu \nu}^{\alpha}$, but also any quantity calculated by using them, will be invariant under Cartan transformations. In fact, the relations (8) provide a prescription to define quantities in a Cartan gauge, starting from their definitions in the related Riemann gauge, in a way that is clearly invariant under Cartan transformations. They are also useful in clarifying the meaning of some physical quantities and equations, whose behavior in a Cartan gauge may appear rather different from their behavior in the Riemann gauge. As we will see later, such is the case, for instance, of the conservation law of matter energy-momentum tensor.

Following the line of thought outlined above, let us consider an arbitrary Cartan gauge $(M, g, \phi)$, in which the scalar torsion and the corresponding contortion will be given, respectively, by

$$
\begin{gathered}
\mathcal{T}_{\mu \nu}^{\alpha}=\frac{1}{2}\left(\delta_{\nu}^{\alpha} \phi_{, \mu}-\delta_{\mu}^{\alpha} \phi_{, \nu}\right), \\
K_{\mu \nu}^{\alpha}=-\frac{1}{2} g^{\alpha \beta}\left(g_{\beta \mu} \phi_{, \nu}-g_{\mu \nu} \phi_{, \beta}\right)=-\frac{1}{2}\left(\delta_{\mu}^{\alpha} \phi_{, \nu}-g_{\mu \nu} \phi^{, \alpha}\right) .
\end{gathered}
$$

\footnotetext{
${ }^{2}$ From now on all quantities referred to the Riemann gauge will denoted by a big hat superscript.
} 
Under these assumptions, it is easy to verify that, in the gauge $(M, g, \phi)$, the Ricci tensor may be written as

$$
R_{\mu \nu}=\widetilde{R}_{\mu \nu}-\left(\widetilde{\nabla}_{\mu} \phi_{, \nu}+\frac{1}{2} g_{\mu \nu} \widetilde{\square} \phi\right)-\frac{1}{2}\left(\phi_{, \mu} \phi_{, \nu}-g_{\mu \nu} \phi_{, \alpha} \phi^{, \alpha}\right),
$$

and the curvature scalar will be given by

$$
R=\widetilde{R}-3 \widetilde{\square} \phi+\frac{3}{2} \phi_{, \alpha} \phi^{, \alpha},
$$

where $\widetilde{\square}=g^{\alpha \beta} \widetilde{\nabla}_{\alpha} \widetilde{\nabla}_{\beta}$. (Note that the Ricci tensor is symmetric, since $\widetilde{\nabla}_{\mu} \phi_{, \nu}=\widetilde{\nabla}_{\nu} \phi_{, \mu}$ ). At this point, let us recall that in a Riemann-Cartan manifold the Bianchi identities are given by $R_{[\beta \mu \nu]}^{\alpha}+\mathcal{T}_{[\beta \mu ; \nu]}^{\alpha}+\mathcal{T}_{[\beta \mu}^{\lambda} \mathcal{T}^{\alpha}{ }_{\nu] \lambda}=0$ and $R_{\beta[\mu \nu ; \sigma]}^{\alpha}+R_{\beta \lambda[\mu}^{\alpha} \mathcal{T}_{\nu \sigma]}^{\lambda}=0$. In the case of scalar torsion, the above identities reduce to $R_{[\beta \mu \nu]}^{\alpha}=0$ and $R_{\beta[\mu \nu ; \lambda]}^{\alpha}+R_{\beta[\mu \nu}^{\alpha} \phi_{, \lambda]}=0$. Finally, the contracted Bianchi identities are given by $\nabla^{\alpha} G_{\alpha \beta}+\phi^{, \alpha} G_{\alpha \beta}=0$, where $G_{\alpha \beta}=R_{\alpha \beta}-\frac{1}{2} g_{\alpha \beta} R$ denotes the Einstein tensor of the Riemann-Cartan space-time manifold. It is clear that the contracted Bianchi identities can be written, alternatively, as

$$
\nabla^{\alpha}\left(e^{\phi} G_{\alpha \beta}\right)=0
$$

Of course all these identities are invariant under Cartan transformations (7). A simple way to show the above results is to start with the Bianchi identities in the Riemann gauge $(M, \widehat{g}, 0)$, where the torsion scalar field is null, and then express them in the Cartan gauge $(M, g, \phi)$ by using the invariant relations (8) between their metrics and connections, namely, $\widehat{g}_{\mu \nu}=e^{-\phi} g_{\mu \nu}$ and $\widehat{\Gamma}_{\mu \nu}^{\alpha}=\Gamma_{\mu \nu}^{\alpha}-\frac{1}{2} \phi_{, \mu} \delta_{\nu}^{\alpha}$.

We assume that physical quantities are invariant under Cartan transformations. Thus, all Cartan gauges related by Cartan transformations may be viewed as different representations of the same fundamental physical entity: the gravitational field, bearing in mind that in an arbitrary Cartan gauge the gravitational field is determined by two independent geometric objects, namely, the metric and the torsion scalar field.

As we know, there are two types of distinguished unparametrized curves, whose properties are defined by the metric and a general linear connection. The first type is the geodesic, also called metric geodesic, which has the property that the space-time interval $d s^{2}=g_{\mu \nu} d x^{\mu} d x^{\nu}$ is an extremum along the curve. Thus, a parametrized time-like curve $x^{\mu}=x^{\mu}(\lambda)$, passing through the events $x^{\mu}(a)$ and $x^{\mu}(b)$, corresponds to a geodesic if and only if it extremizes the time-like space-time interval functional (considering metric assignature -2)

$$
\Delta s=\int_{a}^{b}\left(g_{\mu \nu} \frac{d x^{\mu}}{d \lambda} \frac{d x^{\nu}}{d \lambda}\right)^{\frac{1}{2}} d \lambda .
$$

It should be noted that in spite of the fact that the time-like space-time interval above reduces to the known expression of the proper time in general relativity in the Riemann gauge, there is no reparametrization that could make it invariant under Cartan transformations (77). Therefore, we cannot take $\Delta s$ as the extension to an arbitrary Cartan gauge of the general relativity clock hypothesis, i.e., the assumption that $\Delta s$ measures the proper time interval measured by a clock attached to a test particle. In the next section, we will 
show how to sort out this problem. It is not difficult to verify that the extremization condition of the space-time interval functional leads to the geodesic equations

$$
\frac{d^{2} x^{\alpha}}{d \lambda^{2}}+\left\{\begin{array}{c}
\alpha \\
\mu \nu
\end{array}\right\} \frac{d x^{\mu}}{d \lambda} \frac{d x^{\nu}}{d \lambda}=\frac{d^{2} x^{\alpha}}{d \lambda^{2}}+\left(\Gamma_{\mu \nu}^{\alpha}-K_{\mu \nu}^{\alpha}\right) \frac{d x^{\mu}}{d \lambda} \frac{d x^{\nu}}{d \lambda}=0
$$

where the parameter $\lambda$ is called an affine parameter, since it is fixed up to an affine transformation $\lambda^{\prime}=a \lambda+b$ ( $a, b$ constants). As in general relativity, the parameter $\lambda$ can be identified with the space-time interval $s$ along the curve (up to affine transformations) and, therefore, the quantity $\left(\frac{d s}{d \lambda}\right)^{2}=g_{\mu \nu} \frac{d x^{\mu}}{d \lambda} \frac{d x^{\nu}}{d \lambda}=\left(\frac{1}{a}\right)^{2}$ is constant under Riemann-Cartan parallel transport along the geodesic curve, that is, $\frac{d}{d \lambda}\left(g_{\mu \nu} \frac{d x^{\mu}}{d \lambda} \frac{d x^{\nu}}{d \lambda}\right)=\frac{d x^{\alpha}}{d \lambda} \nabla_{\alpha}\left(g_{\mu \nu} \frac{d x^{\mu}}{d \lambda} \frac{d x^{\nu}}{d \lambda}\right)=$ 0 . Therefore, the equations of a geodesic curve $x^{\mu}=x^{\mu}(s)$, with the space-time interval $s$ being used as a parameter and the tangent vector $v^{\mu}=\frac{d x^{\mu}}{d s}$, are given by

$$
\frac{d^{2} x^{\alpha}}{d s^{2}}+\left\{\begin{array}{c}
\alpha \\
\mu \nu
\end{array}\right\} \frac{d x^{\mu}}{d s} \frac{d x^{\nu}}{d s}=\frac{d^{2} x^{\alpha}}{d s^{2}}+\left(\Gamma_{\mu \nu}^{\alpha}-K_{\mu \nu}^{\alpha}\right) \frac{d x^{\mu}}{d s} \frac{d x^{\nu}}{d s}=0 .
$$

Since the geodesics depend only on the metric, their Cartan transformations (17) involve only the conformal transformations of the metric. Therefore, as in general relativity, they are not invariant under these transformations. The only exception occurs with null geodesics, which require a reparametrization [16].

The second type of unparametrized curves are the so-called auto-parallel curves, also called affine geodesics, since they are curves which parallel-transports their tangent vectors along themselves with respect to the Riemann-Cartan connection [1, 2]. In general the equations of an auto-parallel curve $x^{\mu}=x^{\mu}(\sigma)$, with parameter $\sigma$ and tangent vector $\frac{d x^{\mu}}{d \sigma}$ , are given by

$$
\frac{D}{D \sigma}\left(\frac{d x^{\alpha}}{d \sigma}\right)=\frac{d x^{\mu}}{d \sigma} \nabla_{\mu}\left(\frac{d x^{\alpha}}{d \sigma}\right)=\frac{d^{2} x^{\alpha}}{d \sigma^{2}}+\Gamma_{\mu \nu}^{\alpha} \frac{d x^{\mu}}{d \sigma} \frac{d x^{\nu}}{d \sigma}=h(\sigma) \frac{d x^{\alpha}}{d \sigma},
$$

where $h(\sigma)$ is a function of the curve parameter $\sigma$. The function $h(\sigma)$ can be removed by a reparametrization $\sigma=\sigma(\lambda)$, so that the equations of an auto-parallel curve can be written as

$$
\frac{d^{2} x^{\alpha}}{d \lambda^{2}}+\Gamma_{\mu \nu}^{\alpha} \frac{d x^{\mu}}{d \lambda} \frac{d x^{\nu}}{d \lambda}=0
$$

and the new parameter $\lambda$ is an affine parameter. The affine parameter $\lambda$ of an auto-parallel curve can also be identified with the space-time interval $s$, up to an affine transformation, since the quantity $\left(\frac{d s}{d \lambda}\right)^{2}=g_{\mu \nu} \frac{d x^{\mu}}{d \lambda} \frac{d x^{\nu}}{d \lambda}$ is also constant under Riemann-Cartan parallel transport along the auto-parallel curve. Therefore, the equations of an auto-parallel curve parametrized by the space-time interval, with $v^{\alpha}=\frac{d x^{\alpha}}{d s}$, can be written as

$$
\frac{D v^{\alpha}}{D s}=v^{\mu} \nabla_{\mu} v^{\alpha}=\frac{d v^{\alpha}}{d s}+\Gamma_{\mu \nu}^{\alpha} v^{\mu} v^{\nu}=0
$$

The auto-parallel curves, parametrized either by the space-time interval (19) or by an arbitrary affine parameter (18), are invariant under Cartan transformations (7), although they need to be reparametrized. Due to the identification of the affine parameter with the space-time interval, the necessary reparametrization of the affine parameter must 
be equal to the space-time interval transformation $d \bar{s}=e^{\frac{1}{2} f} d s$, that is, $d \bar{\lambda}=e^{\frac{1}{2} f} d \lambda$. Therefore, they are the natural candidates to describe the motion of test particles and light rays which, are assumed to be invariant under Cartan transformations, as we will see in the next section. In general, geodesics and auto-parallel curves are different unparametrized curves in a Riemann-Cartan space-time manifold, in the sense that geodesics equations cannot be transformed into auto-parallels equations through a reparametrization. There are only two exceptions. The first exception occurs with null geodesics and null auto-parallels, whose equations can be transformed one into another by means of a reparametrization. The second appears when the torsion is totally antisymmetric (axial torsion), since the contortion is also totally antisymmetric and, therefore, geodesic and auto-parallels coincide [17].

Finally, let us remark that geodesics plays a fundamental role in general relativity as well as in most metric theories of gravity. Indeed, an elegant aspect of the geometrization of the gravitational field lies in the geodesics postulate, i.e., the statement that light rays and test particles moving under the influence of gravity alone follow space-time geodesics. Therefore a great deal of information about the motion of particles in a given space-time is promptly available once one knows its geodesics.

The fact that auto-parallel curves are invariant under Cartan gauge transformations and that Riemannian geometry can be seen as a particular case of Riemann-Cartan geometry seems to suggest that it should be possible to express general relativity in a more general geometrical setting, namely, one in which not only the motion of particles and light rays but also the form of the gravitational field equations are also invariant under Cartan gauge transformations.

\section{General Relativity and a New Kind of Invariance}

In this section we will show that it is possible to express general relativity in a RiemannCartan space-time framework. This program will involve several independent steps, consisting basically of the following points: the formulation of the gravitational field equations through a variational principle, the determination of the motion of test particles and light rays, the coupling between matter fields and the gravitational field in a Cartan gauge and, finally, the energy-momentum conservation. We will also include a further requirement: invariance under Cartan transformations. As we will see, the formulation of general relativity in a special class of Riemann-Cartan space-times (those endowed with scalar torsion) entails a complete equivalence among all Cartan gauges. This a consequence of the fact that the mentioned formulation is invariant under Cartan transformations, with the usual Riemannian formulation of general relativity being restored in the Riemann gauge, where the torsion scalar field vanishes. In a certain sense, this reminds us of the gauge freedom exhibited by classical electrodynamics.

To carry out the program outlined above, let us start with the Lagrangian formulation of general relativity. Consider the Einstein-Hilbert Lagrangian $L_{E H}=\sqrt{-\widehat{g}} \widehat{R}$ in a Riemann gauge. In order to obtain the same Lagrangian expressed in an arbitrary Cartan gauge we will make use of (8) . This will lead to $L_{E H}=\sqrt{-g} e^{-2 \phi}\left(e^{\phi} R\right)=\sqrt{-g} e^{-\phi} R$. If we want to include the cosmological constant and matter, then the simplest action that 
can be built under the above conditions is

$$
S=\int d^{4} x \sqrt{-g} e^{-2 \phi}\left(e^{\phi} R+2 \Lambda+\kappa L_{m}\right),
$$

where $L_{m}$ stands for the Lagrangian of the matter fields, $\Lambda$ is the cosmological constant and $\kappa$ is the Einstein's constant. Let us remark that the invariance of the action with respect to Cartan transformations requires the same invariance of both $\Lambda$ and $L_{m}$. The gravitational field equations in a Cartan gauge are obtained by requiring the action (20) to be stationary under arbitrary variations with respect to the two independent elements of the gravitational field, namely, the metric and the torsion scalar field. In this way, we will get the following equations:

$$
G_{\mu \nu}[g, \phi]=\widetilde{G}_{\mu \nu}-\left(\widetilde{\nabla}_{\mu} \phi_{, \nu}-g_{\mu \nu} \tilde{\square} \phi\right)-\frac{1}{2}\left(\phi_{, \mu} \phi_{, \nu}+\frac{1}{2} g_{\mu \nu} \phi_{, \alpha} \phi^{, \alpha}\right)=-\kappa T_{\mu \nu}+g_{\mu \nu} e^{-\phi} \Lambda
$$

and

$$
R[g, \phi]=\widetilde{R}-3 \widetilde{\square} \phi+\frac{3}{2} \phi_{, \alpha} \phi^{, \alpha}=\kappa T-4 e^{-\phi} \Lambda,
$$

where $T=g^{\mu \nu} T_{\mu \nu}$ is the trace of the energy-momentum tensor of matter $T_{\mu \nu} 3$. It should be noted that the above gravitational field equations are not independent, since (22) is just the trace of (21). This is consistent with the fact that we have complete freedom in the choice of the Cartan gauge through the Cartan transformations (7). It also means that one degree of freedom in the pair $\left(g_{\mu \nu}, \phi\right)$ may be viewed as an arbitrary gauge field and not as a dynamical field. Each Cartan gauge corresponds to a different choice of gauge, where either $\phi$ or one of the independent component of $g_{\mu \nu}$ is chosen arbitrarily. Therefore, the gravitational field in a Cartan gauge has the same number of degrees of freedom as the gravitational field in usual formulation of general relativity.

Our next task it to extend Einstein's geodesic postulate to arbitrary Cartan gauges, in an invariant way under Cartan transformations. It will be required that in the Riemann gauge the motion of test particles and light rays in the Riemann gauge should be governed by Einstein's geodesic postulate. It is almost obvious that to complete the required extension we need the following statement: If we represent parametrically a time-like curve as $x^{\mu}=x^{\mu}(\lambda)$, then this curve will represent the world line of a test particle free from all non-gravitational forces, passing through the events $x^{\mu}(a)$ and $x^{\mu}(b)$, if and only if it extremizes the functional

$$
\Delta \tau=\int_{a}^{b}\left(e^{-\phi} g_{\mu \nu} \frac{d x^{\mu}}{d \lambda} \frac{d x^{\nu}}{d \lambda}\right)^{\frac{1}{2}} d \lambda .
$$

Clearly, the above definition is obtained from the special relativistic expression of proper time by using the coupling prescription $\eta_{\mu \nu} \rightarrow e^{-\phi} g_{\mu \nu}$. The right-hand side of this equation is invariant under Cartan transformations and reduces to the known expression of the proper time in general relativity in the Riemann gauge. Therefore, we take $\Delta \tau$, as given

\footnotetext{
${ }^{3}$ Here we are denoting the Einstein tensor and the curvature scalar of the Riemann-Cartan space-time by $G_{\mu \nu}[g, \phi]$ and $R[g, \phi]$, respectively, to emphasize that they depend both on the metric $g$ and on the torsion scalar field $\phi$. Whenever necessary, the same notation will be used for other quantities of the Riemann-Cartan space-time.
} 
above, as the extension to an arbitrary Cartan gauge of the clock hypothesis, i.e., the assumption that $\Delta \tau$ measures the proper time measured by a clock attached to the test particle. Note that, in a Cartan gauge, the proper time depends on both fundamental elements of the gravitational field, i.e., $\left(g_{\mu \nu}, \phi\right)$. It is not difficult to verify that the extremization condition of the proper time functional (23) leads to the following equations of motion of a test particle moving only under the gravitational field influence

$$
\frac{d^{2} x^{\alpha}}{d \lambda^{2}}+\left\{\begin{array}{c}
\alpha \nu \\
\mu \nu
\end{array} \frac{d x^{\mu}}{d \lambda} \frac{d x^{\nu}}{d \lambda}+\frac{1}{2} \phi^{, \beta} g_{\mu \nu} \frac{d x^{\mu}}{d \lambda} \frac{d x^{\nu}}{d \lambda}-\phi_{, \nu} \frac{d x^{\nu}}{d \lambda} \frac{d x^{\alpha}}{d \lambda}=0 .\right.
$$

Let us recall that in the derivation of the above equations it was considered the fact that the quantity $\left(\frac{d \tau}{d \lambda}\right)^{2}=e^{-\phi} g_{\mu \nu} \frac{d x^{\mu}}{d \lambda} \frac{d x^{\nu}}{d \lambda}$ is constant under Riemann-Cartan parallel transport along the curve, i.e., that $\frac{d x^{\mu}}{d \lambda} \nabla_{\mu}\left(e^{-\phi} g_{\mu \nu} \frac{d x^{\mu}}{d \lambda} \frac{d x^{\nu}}{d \lambda}\right)=0$, which allows the identification of $\lambda$ with the proper time $\tau$, up to an affine transformation. Substituting the expression for the contortion given by (10) in (24) we have

$$
u^{\mu} \nabla_{\mu} u^{\alpha}-\frac{1}{2} \frac{d \phi}{d \tau} u^{\alpha}=\frac{d u^{\alpha}}{d \tau}+\Gamma_{\mu \nu}^{\alpha} u^{\mu} u^{\nu}-\frac{1}{2} \frac{d \phi}{d \tau} u^{\alpha}=0,
$$

where $u^{\beta}=\frac{d x^{\beta}}{d \tau}$ is the 4 -velocity of the test particle and $\frac{d \phi}{d \tau}=\phi_{, \mu} u^{\mu}$ is the variation of the torsion scalar field along the curve. These are the equations of the auto-parallel curves (17) defined by the Riemann-Cartan connection, parametrized with the proper time (23). They are invariant under Cartan transformations and reduce to the known geodesic equations in general relativity in the Riemann gauge. On the other hand, the proper time is not an affine parameter. Nevertheless, the standard form of the equations of auto-parallel curves (19), with the space-time interval as an affine parameter, can be obtained from (25) through a reparametrization $\tau=\tau(s)$ given by $d \tau=e^{-\frac{1}{2} \phi} d s$, which leads to $u^{\mu} \nabla_{\mu} u^{\alpha}-\frac{1}{2} \frac{d \phi}{d \tau} u^{\alpha}=e^{\phi} v^{\mu} \nabla_{\mu} v^{\alpha}=0$, where $v^{\alpha}=\frac{d x^{\alpha}}{d s}$. Therefore, the extension of the geodesic postulate to a Cartan gauge by requiring the proper time functional (23) to be an extremum along the path of a test particle free from all non-gravitational forces, is equivalent to postulating that the motion of a test particle on the gravitational field must follow auto-parallel time-like curves defined by the Riemann-Cartan connection.

Considering the unusual form of the equations of motion (25) of a test particle moving only under the gravitational field influence, written in terms of the proper time $\tau$, it is valuable to make some comparisons between the proper time $\tau$ and the space-time interval $s$. The proper-time (23) is invariant under Cartan transformations, but it is not an affine parameter for test particle paths, that is, the auto-parallel time-like curves. On the other hand, the space-time interval is an affine parameter for the test particles paths, but it is not invariant under Cartan transformations. At this point it is important to note that the tangent vector to the time-like auto-parallel curves may have different behaviors, depending on whether the space-time interval or the proper time is used as parameter. When the parameter is the space-time interval, the norm of the tangent vector $v^{\alpha}=\frac{d x^{\alpha}}{d s}$ is constant since $g_{\alpha \beta} v^{\alpha} v^{\beta}=1$ along the time-like auto-parallel curve. Indeed, we have $\frac{d}{d s}\left(g_{\alpha \beta} v^{\alpha} v^{\beta}\right)=0$, which is a consequence of the equations (19) of the auto-parallel curve with the space-time interval as affine parameter. On the other hand, when the parameter is the proper time, the norm of the 4 -velocity vector $u^{\alpha}=\frac{d x^{\alpha}}{d \tau}$ depends on the value of the torsion scalar field, since $e^{-\phi} g_{\alpha \beta} u^{\alpha} u^{\beta}=1$ is constant along the time-like auto-parallel 
curve, according to $\frac{d}{d \tau}\left(e^{-\phi} g_{\alpha \beta} u^{\alpha} u^{\beta}\right)=u^{\mu} \nabla_{\mu}\left(e^{-\phi} g_{\alpha \beta} u^{\alpha} u^{\beta}\right)=0$, as a consequence of the equations (25) of the auto-parallel curve in which the affine parameter is the proper time. We see, then, that the 4 -velocity $u^{\alpha}=\frac{d x^{\alpha}}{d \tau}$ differs from the tangent vector $v^{\alpha}=\frac{d x^{\alpha}}{d s}$ by a factor due to the torsion scalar field, that is, $u^{\alpha}=e^{-\frac{1}{2} \phi} v^{\alpha}$, since the proper time in a Cartan gauge depends on both the metric and the torsion scalar field. The space-time interval, in turn, depends only on the metric. Owing to this correction, the norm of the 4velocity depends on the torsion scalar field through the invariant expression $g_{\alpha \beta} u^{\alpha} u^{\beta}=e^{\phi}$. The norm of the 4 -velocity will be constant only in the special case in which the torsion scalar field is constant along the auto-parallel curve, that is, when $\frac{d \phi}{d \tau}=\phi_{, \mu} u^{\mu}=0$. Indeed, in this case we have $\frac{d}{d \tau}\left(g_{\alpha \beta} u^{\alpha} u^{\beta}\right)=u^{\mu} \nabla_{\mu}\left(g_{\alpha \beta} u^{\alpha} u^{\beta}\right)=e^{\phi} \frac{d \phi}{d \tau}=0$.

A simple way to show the above results is to start from the Riemann gauge $(M, \widehat{g}, 0)$, where $\widehat{\phi}=0$, and then change to a Cartan gauge $(M, g, \phi)$ by using the invariant relations (8), that is, $\widehat{g}_{\mu \nu}=e^{-\phi} g_{\mu \nu}$ and $\widehat{\Gamma}_{\mu \nu}^{\alpha}=\Gamma_{\mu \nu}^{\alpha}-\frac{1}{2} \phi_{, \mu} \delta_{\nu}^{\alpha}$. Thus, let us consider a test particle moving only under the influence of the gravitational field. Considering that the 4-velocity $u^{\alpha}=\frac{d x^{\alpha}}{d \tau}$ is an invariant (the coordinates and the proper time are both invariant) and that the norm of the 4-velocity $\widehat{u}^{\alpha}$ in the Riemann gauge is constant, it follows that the norm of the 4-velocity in a Cartan gauge depends on the torsion scalar field according to the invariant relation $\widehat{g}_{\alpha \beta} \widehat{u}^{\alpha} \widehat{u}^{\beta}=e^{-\phi} g_{\alpha \beta} u^{\alpha} u^{\beta}=1$. Moreover, since in the Riemann gauge the 4-acceleration $\widehat{a}^{\beta}$ vanishes, in accordance with the principle of equivalence, it follows that the test particle moves, in a Cartan gauge, along a time-like auto-parallel curve with the proper time as an affine parameter. Indeed, we have the invariant relations $\widehat{a}^{\beta}=\widehat{u}^{\mu} \widehat{\nabla}_{\mu} \widehat{u}^{\beta}=u^{\mu} \nabla_{\mu} u^{\beta}-\frac{1}{2} \frac{d \phi}{d \tau} u^{\beta}=e^{\phi} v^{\mu} \nabla_{\mu} v^{\alpha}=0$. These are the same equations of motion (25) that were previously obtained from the extension of the geodesic postulate and the definition of proper time in a Cartan gauge. Therefore, it seems natural to define the 4 -acceleration in a Cartan gauge as the invariant quantity

$$
a^{\beta}=u^{\mu} \nabla_{\mu} u^{\beta}-\frac{1}{2} \frac{d \phi}{d \tau} u^{\beta}=e^{\phi} v^{\mu} \nabla_{\mu} v^{\alpha} .
$$

Two comments are in order. First, as a consequence of the dependence of the norm of the 4 -velocity on the torsion scalar field, given by $g_{\alpha \beta} u^{\alpha} u^{\beta}=e^{\phi}$, it follows that the invariant relation $g_{\alpha \beta} u^{\alpha} a^{\beta}=0$ is satisfied. Second, the condition $a^{\beta}=0$, valid for a test particle moving only under the gravitational field influence, is a consequence of the equivalence principle, and means that the test particle follows a time-like auto-parallel curve parametrized with proper time.

As we know, the geodesic postulate in general relativity is a statement which rules not only to the motion of test particles, but also to the propagation of light rays in spacetime. Because the path of light rays are null curves, one cannot use the proper time as a parameter to describe them. On the other hand, it seems natural to assume that light rays follow null auto-parallel curves. Thus, these curves cannot be defined in terms of the proper time functional (23), which is identically null for these curves. They must be characterized instead by their behavior with respect to the Riemann-Cartan parallel transport. We will extend this postulate by simply assuming that light rays follow null auto-parallel curves defined by the Riemann-Cartan connection. The equations of motion of light rays are the equations of null auto-parallel curves $x^{\mu}=x^{\mu}(\lambda)$ with affine parameter $\lambda$. They can be obtained from the auto-parallel equations (18) by requiring the tangent 
vector to be a null vector, that is, $g_{\mu \nu} \frac{d x^{\mu}}{d \lambda} \frac{d x^{\nu}}{d \lambda}=0$, and are given by

$$
\frac{d^{2} x^{\alpha}}{d \lambda^{2}}+\left\{\begin{array}{c}
\alpha \\
\mu \nu
\end{array}\right\} \frac{d x^{\mu}}{d \lambda} \frac{d x^{\nu}}{d \lambda}-\frac{1}{2} \phi_{, \nu} \frac{d x^{\nu}}{d \lambda} \frac{d x^{\alpha}}{d \lambda}=0
$$

which, through a reparametrization $\lambda=\lambda(\sigma)$ given by $d \lambda=e^{\frac{1}{2} \phi} d \sigma$, are equal to the equations of a null (metric) geodesic (15) with affine parameter $\sigma$, that is, $\frac{d^{2} x^{\alpha}}{d \sigma^{2}}+\left\{\begin{array}{c}\alpha \\ \mu \nu\end{array}\right\} \frac{d x^{\mu}}{d \sigma} \frac{d x^{\nu}}{d \sigma}=0$. Therefore, in a Riemann-Cartan space-time with scalar torsion, null geodesics and null auto-parallel curves coincide, up to a reparametrization, and are given by the same unparametrized null curves. Consequently, in the Riemann-Cartan space-time, these curves define the same light cone and the same local causal structure. Furthermore, according to this result, the local causal structure of space-time in an arbitrary Cartan gauge is determined only by the metric structure (with an indirect influence of the torsion through the gravitational field equations).

Note that the light cone structure of the space-time does not change under Cartan transformations, since it is well known that the equations of null geodesics are preserved under conformal transformations (11), although one needs to reparametrize the curves in the new gauge [16]. In the case of Riemann-Cartan space-times with scalar torsion, the invariance of the equations of null auto-parallel curves also needs a reparametrization, since the connection components $\Gamma_{\alpha \beta}^{\mu}$ change under Cartan transformations (7) according to (2). As a consequence, the causal structure of space-time remains unchanged in all Cartan gauges.

The following step in our formulation has a subtle point in the framework of nonRiemannian space-times. It is related to the question of how to define the coupling between the matter fields and the gravitational field in a Cartan gauge. Clearly, the choice of this coupling must be guided by the requirement of invariance of the field equations with respect to Cartan transformations. It will be assumed that the matter Lagrangian $L_{m}$ in a Cartan gauge generally depends on the two independent fundamental elements of the gravitational field $g_{\mu \nu}$ and $\phi$ and on the matter fields, here generically denoted by $\xi$. The Lagrangian $L_{m}[g, \phi, \xi, \nabla \xi]$ of the matter field $\xi$ in a Cartan gauge can be obtained from the Lagrangian of the matter field in special relativity theory $L_{m}^{s r}=L_{m}^{s r}[\eta, \xi, \partial \xi]$, where $\eta=\left(\eta_{\mu \nu}\right)$ is the Minkowski metric, through the prescription

$$
\eta_{\mu \nu} \rightarrow \widehat{g}_{\mu \nu}, \partial_{\mu} \rightarrow \widehat{\nabla}_{\mu}
$$

where $\widehat{g}_{\mu \nu}=e^{-\phi} g_{\mu \nu}\left(\widehat{g}^{\alpha \beta}=e^{\phi} g^{\alpha \beta}\right)$ and $\widehat{\nabla}_{\mu}$ is the covariant derivative with respect to the connection $\widehat{\Gamma}_{\mu \nu}^{\alpha}=\Gamma_{\mu \nu}^{\alpha}-\frac{1}{2} \phi_{, \mu} \delta^{\alpha}{ }_{\nu}$. Therefore, given the Lagrangian of matter in special relativity theory $L_{m}^{s r}=L_{m}^{s r}[\eta, \xi, \partial \xi]$, we define the coupling between the matter fields and the two independent elements of the gravitational field $\left(g_{\mu \nu}, \phi\right)$ in a Cartan gauge by the rule

$$
L_{m}^{s r}[\eta, \xi, \partial \xi] \rightarrow L_{m}[g, \phi, \xi, \nabla \xi]=L_{m}^{s r}[\widehat{g}, \phi, \xi, \widehat{\nabla} \xi] .
$$

Besides, in order to assure the invariance of the coupling procedure and the matter Lagrangian $L_{m}[g, \phi, \xi, \nabla \xi]$ with respect to Cartan transformations (7), it is necessary to assume that the matter field $\xi$ is also invariant, since by the above rule $L_{m}[g, \phi, \xi, \nabla \xi]$ 
depends on $g_{\mu \nu}$ and $\phi$ only through the invariant combinations $e^{-\phi} g_{\mu \nu}$ and $e^{\phi} g^{\mu \nu}$. Furthermore, it follows that the part of the action (20) that is responsible for the coupling of matter with the gravitational field is also invariant. Finally, this prescription reduces to the principle of minimal coupling adopted in general relativity when we set $\phi=0$, that is, in the Riemann gauge.

The final step is closely related to the previous one, since it is the definition of the energy-momentum tensor of the matter fields and the formulation of its conservation law. Similarly to the Einstein equations in a Riemann gauge, the gravitational field equations $G_{\mu \nu}=-k T_{\mu \nu}$ (21) in a Cartan gauge has a geometric quantity $G_{\mu \nu}$ on the left-hand side and a non-geometric quantity $T_{\mu \nu}$ on the right-hand side. On the other hand, the invariance of the field equations (21) under Cartan transformations require that both $G_{\mu \nu}$ and $T_{\mu \nu}$ have the same transformation laws, in spite of being of completely different nature. This condition put several restrictions to the possible choices on the coupling between gravitation and matter and, consequently, on the forms of both the Lagrangian and the energy-momentum tensor of matter fields. A important restriction is the requirement of invariance of the covariant components $T_{\mu \nu}$ with respect to Cartan transformations, since $G_{\mu \nu}$ is already invariant under these transformations. However, as we will show in the following, it is possible to give a definition of the energy-momentum tensor, based on the previous definition of the matter Lagrangian in a Cartan gauge, which satisfies these conditions and is consistent with all previous steps.

The Lagrangian $L_{m}[g, \phi, \xi, \nabla \xi]$ of the matter field, defined in an arbitrary Cartan gauge $(M, g, \phi)$ by the rule (29), has a remarkable property: it depends on the basic elements of the gravitational field in a Cartan gauge, $g_{\mu \nu}$ and $\phi$, only through the invariant combinations $e^{-\phi} g_{\mu \nu}$ and $e^{\phi} g^{\mu \nu}$. Therefore, it is natural to define the energy-momentum tensor $T_{\mu \nu}[g, \phi, \xi, \nabla \xi]$ in a Cartan gauge by the formula

$$
\delta S_{m}=\delta \int d^{4} x \sqrt{-g} e^{-2 \phi} L_{m}[g, \phi, \xi, \nabla \xi]=\int d^{4} x \sqrt{-g} e^{-2 \phi} T_{\mu \nu}[g, \phi, \xi, \nabla \xi] \delta\left(e^{\phi} g^{\mu \nu}\right),
$$

where the variation must be carried out with respect to both $g_{\mu \nu}$ and $\phi$. This definition is not only invariant under Cartan transformations (7), but also reduces to general relativity definition of the energy-momentum tensor in the Riemann gauge. It should be mentioned that the above definition is invariant under Cartan transformations, since besides the invariants $L_{m}[g, \phi, \xi, \nabla \xi]$ and $e^{\phi} g^{\mu \nu}$ there is also the invariant quantity $d \widehat{V}=\sqrt{-\widehat{g}} d^{4} x=$ $\sqrt{-g} e^{-2 \phi} d^{4} x$.

We would like to conclude this section with a brief comment on the form of the energy-momentum conservation law in a arbitrary Cartan gauge. We start with the gravitational field equations in the Riemann gauge $(M, \widehat{g}, \widehat{\phi}=0)$, given by $\widehat{G}_{\mu \nu}[\widehat{g}, 0]=$ $-\kappa \widehat{T}_{\mu \nu}[\widehat{g}, 0, \xi, \widehat{\nabla} \xi]$, where the Einstein tensor $\widehat{G}_{\mu \nu}[\widehat{g}, 0]$ is divergenceless. Due to this property, it follows from (21) that the energy-momentum conservation law in the Riemann gauge $(M, \widehat{g}, \widehat{\phi}=0)$ is given by

$$
\widehat{\nabla}^{\mu} \widehat{T}_{\mu \nu}=\widehat{g}^{\mu \alpha} \widehat{\nabla}_{\alpha} \widehat{T}_{\mu \nu}=0
$$

This shows that, in the Riemann gauge $(M, \widehat{g}, \widehat{\phi}=0)$, the matter field interacts only with the space-time metric, as it should be. On the other hand, we obtain the same gravitational field equations (21), i.e., $G_{\mu \nu}[g, \phi]=-\kappa T_{\mu \nu}[g, \phi, \xi, \nabla \xi]$, if we go to an arbitrary 
Cartan gauge $\left(M, g=e^{\phi} \widehat{g}, \phi\right)$. However, now the Einstein tensor $G_{\mu \nu}[g, \phi]$ is not divergenceless. Indeed, a straightforward calculation shows that $\widehat{\nabla}^{\alpha} \widehat{G}_{\alpha \mu}=\nabla^{\alpha}\left(e^{\phi} G_{\alpha \mu}\right)=0$, in accordance with the contracted Bianchi identities (13). Therefore, it follows that the energy-momentum conservation law in the Cartan gauge $(M, g, \phi)$ takes the form $\widehat{\nabla}^{\alpha} \widehat{T}_{\alpha \mu}=\nabla^{\alpha}\left(e^{\phi} T_{\alpha \mu}\right)=0$, whence

$$
\nabla^{\alpha}\left(e^{\phi} T_{\alpha \mu}\right)=e^{\phi}\left(\nabla^{\alpha} T_{\alpha \mu}+\phi^{, \alpha} T_{\alpha \mu}\right)=e^{\phi}\left(\widetilde{\nabla}^{\alpha} T_{\alpha \mu}-\phi^{, \alpha} T_{\alpha \mu}+\frac{1}{2} \phi_{, \mu} T\right)=0,
$$

since $\nabla^{\alpha} T_{\alpha \mu}=\widetilde{\nabla}^{\alpha} T_{\alpha \mu}-2 \phi^{, \alpha} T_{\alpha \mu}+\frac{1}{2} \phi_{, \mu} T$. Note that the law of conservation of the energy-momentum tensor (32) is a consequence of the Bianchi identities (13), not only in the Riemann gauge but also in any Cartan gauge. Furthermore, it is not difficult to verify that the above equations are invariant under the Cartan transformations (17). From the invariance of both the gravitational field equations $G_{\mu \nu}=-\kappa T_{\mu \nu}$ (21) and the Bianchi identities $\nabla^{\alpha}\left(e^{\phi} G_{\alpha \mu}\right)=0$, we obtain that in the Cartan gauge $(M, g, \phi)$ the law of conservation of the energy-momentum tensor is given by $\nabla^{\alpha}\left(e^{\phi} T_{\alpha \mu}\right)=0$.

The presence of non-vanishing terms on the right-hand side of (32) may led someone to think that the energy-momentum is not conserved in a Cartan gauge. However, we must remember that the torsion scalar field $\phi$ is an intrinsic element of the gravitational field in the Riemann-Cartan space-time and should necessarily appear in any equation describing the behavior of matter in any Cartan gauge. This explain the presence of $\phi$ coupled with $T_{\mu \nu}$ in (32) and the apparent violation of the energy-momentum conservation law. Note that the familiar energy-momentum conservation equations of general relativity are recovered when $\phi=0$, that is, in the Riemann gauge.

It is important to pay attention to the fact that all physical quantities are invariant under Cartan transformations and only in the Riemann frame they are given by the familiar general relativity expressions. In a Cartan frame, on the other hand, they are given by more general expressions involving not only the metric but also the torsion scalar and require a careful interpretation to deal with the Riemann-Cartan framework. The unique Riemann gauge and all the Cartan gauges are equivalent from the experimental point of view, since any physical quantity has the same numerical value in any of them.

Let us now apply the above results to describe the interaction of gravity with a perfect fluid in an arbitrary Cartan frame. The energy-momentum tensor of a perfect fluid in special relativity is defined by $T^{\mu \nu}=\left(\rho c^{2}+p\right) u^{\mu} u^{\nu}-p \eta^{\mu \nu}$, where $\rho, p$ and $u^{\mu}=\frac{d x^{\mu}}{d \tau}$ denotes, respectively, the proper density, the pressure and the 4 -velocity fields. We now need to express $T^{\mu \nu}$ in an arbitrary Cartan gauge. Rewriting this expression as $T_{\mu \nu}=$ $\left(\rho c^{2}+p\right) \eta_{\mu \alpha} \eta_{\nu \gamma} u^{\alpha} u^{\gamma}-p \eta_{\mu \nu}$ and following the coupling prescription $\eta_{\mu \nu} \rightarrow e^{-\phi} g_{\mu \nu}$, we obtain

$$
T_{\mu \nu}=\left(\rho c^{2}+p\right) e^{-2 \phi} g_{\mu \alpha} g_{\nu \gamma} u^{\alpha} u^{\gamma}-p e^{-\phi} g_{\mu \nu},
$$

which is the desired expression of the energy-momentum tensor of a perfect fluid in an arbitrary Cartan gauge. It is worth remembering that, due to the field equations (21), $T_{\mu \nu}$, as given by the above equation, is required to be invariant under Cartan transformations, since the Einstein tensor $G_{\mu \nu}$ is invariant. Therefore, it follows that $\rho$ and $p$ must be regarded as invariant quantities, since $u^{\alpha}=\frac{d x^{\alpha}}{d \tau}$ and $e^{-\phi} g_{\mu \nu}$ are invariant. The same conclusion follows from the invariant relations $\widehat{T}_{\mu \nu} \widehat{u}^{\mu} \widehat{u}^{\nu}=T_{\mu \nu} u^{\mu} u^{\nu}=\rho c^{2}$ and 
$\widehat{T}=\widehat{T}_{\mu \nu} \widehat{g}^{\mu \nu}=T_{\mu \nu} e^{\phi} g^{\mu \nu}=e^{\phi} T=\left(\rho c^{2}-3 p\right)$.

Let us now take a look at the motion of the fluid particles in an arbitrary Cartan gauge. Considering, as source of the gravitational field, a pressureless perfect fluid (dust), it is straightforward to show that the fluid particles move along auto-parallel time-like curves as a consequence of the energy-momentum conservation law (32). This is in accordance with the equivalence principle, now seen from the point of view of a Cartan frame, through the extension of the geodesic postulate to a Cartan gauge.

Finally, let us consider the coupling between the gravitational and the electromagnetic field in a Cartan gauge. In special relativity, the Lagrangian of the electromagnetic field $F_{\mu \nu}=A_{\mu, \nu}-A_{\nu, \mu}$ is given by $L_{e m}^{s r}=\frac{1}{2} \eta^{\alpha \mu} \eta^{\beta \nu} F_{\alpha \beta} F_{\mu \nu}$ [21]. Applying the coupling prescription (28), i.e., $\eta_{\mu \nu} \rightarrow \widehat{g}_{\mu \nu}$ and $\partial_{\mu} \rightarrow \widehat{\nabla}_{\mu}$, we obtain that the Lagrangian in a Cartan gauge is given by $L_{e m}=\frac{1}{2} e^{2 \phi} g^{\alpha \mu} g^{\beta \nu} \widehat{F}_{\alpha \beta} \widehat{F}_{\mu \nu}$, where the electromagnetic field is defined by $\widehat{F}_{\mu \nu}=\widehat{\nabla}_{\nu} \widehat{A}_{\mu}-\widehat{\nabla}_{\mu} \widehat{A}_{\nu}$. As we have seen, the assumption of invariance of the electromagnetic potential under Cartan transformations $\widehat{A}_{\mu}=A_{\mu}$, also assures the invariance of the Lagrangian of the electromagnetic field. At the first sight, due to the above definition, it seems that the electromagnetic field couples with the torsion scalar field in the Cartan gauge, but this is not true. According to the coupling procedure (28), the covariant derivative $\widehat{\nabla}_{\nu} \widehat{A}_{\mu}$ of the electromagnetic potential used to define $\widehat{F}_{\mu \nu}$ is given by $\widehat{\nabla}_{\nu} A_{\mu}=\widetilde{\nabla}_{\nu} A_{\mu}+\frac{1}{2}\left(\phi_{, \nu} A_{\mu}+\phi_{, \mu} A_{\nu}\right)-\frac{1}{2} g_{\mu \nu} \phi^{, \alpha} A_{\alpha}$, and, thus, it follows that

$$
\widehat{F}_{\mu \nu}=\widehat{\nabla}_{\nu} \widehat{A}_{\mu}-\widehat{\nabla}_{\mu} \widehat{A}_{\nu}=F_{\mu \nu}=A_{\mu, \nu}-A_{\nu, \mu} .
$$

Because the electromagnetic field does not couple with the torsion scalar field, the Lagrangian of the electromagnetic field in a Cartan gauge will be given by

$$
L_{e m}=\frac{1}{2} e^{2 \phi} F^{\mu \nu} F_{\mu \nu}
$$

where $F^{\mu \nu}=g^{\alpha \mu} g^{\beta \nu} F_{\alpha \beta}$. On the other hand, since $\widehat{F}_{\mu \nu}=F_{\mu \nu}$, it follows that $L_{e m}$ is also invariant under the gauge transformations of the electromagnetic potential $A_{\mu}^{\prime}=A_{\mu}+f_{, \mu}$. Now, by applying the definition of the energy-momentum tensor (30), we find that the electromagnetic field energy-momentum tensor in a Cartan gauge is given by

$$
T_{\mu \nu}=e^{\phi}\left(F_{\mu}{ }^{\alpha} F_{\alpha \nu}+\frac{1}{2} g_{\mu \nu} F^{\alpha \beta} F_{\alpha \beta}\right)
$$

whose conservation law, according to (32), is given by

$$
\widetilde{\nabla}^{\mu} T_{\mu \nu}-\phi^{, \mu} T_{\mu \nu}+\frac{1}{2} \phi_{, \nu} T=\widetilde{\nabla}^{\mu}\left(F_{\mu}{ }^{\alpha} F_{\alpha \nu}+\frac{1}{2} g_{\mu \nu} F^{\alpha \beta} F_{\alpha \beta}\right)=0,
$$

and does not involve the torsion scalar field as well. Moreover, similarly to general relativity, we obtain that light rays move on null metric geodesic in a Cartan gauge, as a consequence of the conservation laws (37). This is in accordance with the equivalence principle and the extension of the geodesic postulate for the motion of light rays in a Cartan gauge. This seems to complete our program of formulating general relativity in a geometrical setting that exhibits a new kind of invariance, namely, that with respect to Cartan transformations. 


\section{The Newtonian limit in a general Cartan gauge}

In order to gain some insight into the meaning of this new representation of general relativity developed in the previous sections, let us now proceed to examine the Newtonian limit of general relativity in an arbitrary Cartan gauge $(M, g, \phi)$. As we know, a metric theory of gravity is said to possess a Newtonian limit in the non-relativistic weak-field regime if one can derive Newton's second law from the geodesic equations and the Poisson equation from the gravitational field equations. Let us see how general relativity, when expressed in a form that is invariant under Cartan gauge transformations, fulfills these requirements. The method we will employ here to treat this problem is standard and can be found in most textbooks on general relativity (see, for instance, [21]). Since in Newtonian mechanics the space geometry is Euclidean, a weak gravitational field in a geometric theory of gravity should manifest itself as a metric phenomenon through a slight perturbation of the Minkowskian space-time metric. Thus, we consider a timeindependent metric tensor of the form

$$
g_{\mu \nu}=\eta_{\mu \nu}+\epsilon h_{\mu \nu}
$$

where $n_{\mu \nu}$ is the Minkowski metric (with assignature -2), $\epsilon$ is a small parameter $\left(\epsilon^{2} \ll \epsilon\right)$ and the term $\epsilon h_{\mu \nu}$ represents a very small time-independent perturbation due to the presence of some matter configuration. Because we are working in the non-relativistic regime we will suppose that the velocity $V$ of the particle along its path is much less than $c$, so that the parameter $\beta=\frac{V}{c}$ will be regarded as very small; hence in our calculations only first-order terms in $\epsilon$ and $\beta$ will be retained. The same kind of approximation will be assumed with respect to the torsion scalar field $\phi$, which will be supposed to be static and small, i.e., of the same order as $\epsilon$, and, in order to emphasize this fact, we will write $\phi=\epsilon \varphi$, where $\varphi$ is a finite function. Then, adopting the usual Minkowskian coordinates of special relativity we can write the line element defined by (38) as $d s^{2}=\left(\eta_{\mu \nu}-\epsilon h_{\mu \nu}\right) d x^{\mu} d x^{\nu}$, which leads, in our approximation, to

$$
\frac{d \tau}{c d t}=e^{-\frac{1}{2} \phi} \frac{d s}{c d t} \cong\left(1-\frac{1}{2} \epsilon \varphi\right)\left(1+\frac{1}{2} \epsilon h_{00}\right) \cong\left[1+\frac{1}{2} \epsilon\left(h_{00}-\varphi\right)\right],
$$

where $\tau$ is the proper time defined by (23), whose inverse is given by

$$
c \frac{d t}{d \tau} \cong\left[1-\frac{1}{2} \epsilon\left(h_{00}-\varphi\right)\right]
$$

We will now consider, in the same approximation, the invariant affine geodesic equations (25) , i.e.,

$$
\frac{d^{2} x^{\mu}}{d \tau^{2}}+\Gamma_{\alpha \beta}^{\mu} \frac{d x^{\alpha}}{d \tau} \frac{d x^{\beta}}{d \tau}=\frac{1}{2} \frac{d \phi}{d \tau} \frac{d x^{\mu}}{d \tau}
$$

which, by changing the parameter from the proper time $\tau$ to time coordinate $t$, can be written as

$$
\left(\frac{d t}{d \tau}\right)^{2}\left(\frac{d^{2} x^{\mu}}{d t^{2}}+\Gamma_{\alpha \beta}^{\mu} \frac{d x^{\alpha}}{d t} \frac{d x^{\beta}}{d t}\right)=\left[-\frac{d}{d t}\left(\frac{d t}{d \tau}\right)+\frac{1}{2} \frac{d \phi}{d t} \frac{d t}{d \tau}\right]\left(\frac{d t}{d \tau}\right) \frac{d x^{\mu}}{d t} .
$$


Since both $\frac{d t}{d \tau}$, given by (40), and $\phi=\epsilon \varphi$ do not depend on the time coordinate, the right hand side of the above equations is identically zero, and we obtain that

$$
\frac{d^{2} x^{\alpha}}{d t^{2}}+\Gamma_{\mu \nu}^{\alpha} \frac{d x^{\mu}}{d t} \frac{d x^{\nu}}{d t}=0
$$

recalling that the symbol $\Gamma_{\alpha \beta}^{\mu}$ designates the components of the Riemann-Cartan affine connection with a scalar torsion. From the Christoffel symbols and the contortion tensor (10) it is easy to verify that, to first order in $\epsilon$, we have

$$
\Gamma_{\mu \nu}^{\alpha}=\frac{\epsilon}{2} \eta^{\alpha \lambda}\left(h_{\lambda \nu, \mu}+h_{\mu \lambda, \nu}-h_{\mu \nu, \lambda}\right)-\frac{\epsilon}{2} \eta^{\alpha \lambda}\left(\eta_{\lambda \mu} \varphi_{, \nu}-\eta_{\mu \nu} \varphi_{, \lambda}\right) .
$$

It is not difficult to see that, unless $\mu=\nu=0$, the product $\Gamma_{\mu \nu}^{\alpha} \frac{d x^{\mu}}{d t} \frac{d x^{\nu}}{d t}$ is of order $\epsilon \beta$ or higher. In this way, the affine geodesic equations (42) become, to first order in $\epsilon$ and $\beta$,

$$
\frac{d^{2} x^{\alpha}}{d t^{2}}+c^{2} \Gamma_{00}^{\alpha}=0
$$

Clearly for $\alpha=0$ the equations (44) reduces to an identity. On the other hand, if $\alpha$ is a spatial index $i$, a simple calculation yields $\Gamma_{00}^{i}=-\frac{\epsilon}{2} \eta^{i k} \frac{\partial}{\partial x^{k}}\left(h_{00}-\varphi\right)=\frac{\epsilon}{2} \delta^{i k} \frac{\partial}{\partial x^{k}}\left(h_{00}-\right.$ $\varphi)$, hence the affine geodesic equations (41) in this approximation become, in threedimensional vector notation,

$$
\frac{d \vec{V}}{d t}=-\frac{\epsilon c^{2}}{2} \vec{\nabla}\left(h_{00}-\varphi\right),
$$

which is simply Newton's equations of motion in a classical gravitational field, provided we identify the scalar gravitational potential with

$$
U=\frac{\epsilon c^{2}}{2}\left(h_{00}-\varphi\right)
$$

Note the presence of the torsion scalar field $\phi=\epsilon \varphi$ in the above equation. It means that it is the combination $\left(\epsilon h_{00}-\phi\right)$ that represents the Newtonian potential in a Cartan gauge.

Let us now turn our attention to the Newtonian limit of the gravitational field equations. For this purpose, it will be convenient to rewrite the field equations (21) with $\Lambda=0$ in the form

$$
R_{\mu \nu}[g, \phi]=\widetilde{R}_{\mu \nu}-\left(\widetilde{\nabla}_{\mu} \phi_{, \nu}+\frac{1}{2} g_{\mu \nu} \widetilde{\square} \phi\right)-\frac{1}{2}\left(\phi_{, \mu} \phi_{, \nu}-g_{\mu \nu} \phi_{, \alpha} \phi^{, \alpha}\right)=-\kappa\left(T_{\mu \nu}-\frac{1}{2} g_{\mu \nu} T\right) .
$$

In the weak-field approximation, i.e., when $g_{\mu \nu}=\eta_{\mu \nu}+\epsilon h_{\mu \nu}$ and $\phi=\epsilon \varphi$, it is easy to show that, to first order in $\epsilon$, we have $\widetilde{R}_{00}=-\frac{1}{2} \nabla^{2} \epsilon h_{00}$ and $\widetilde{\square} \phi=-\nabla^{2} \epsilon \varphi$, where $\nabla^{2}$ denotes the Laplacian operator calculated with the Minkowski metric. On the other hand, because we are assuming a static regime $\partial_{0} \phi=0$, the equation (47) for $\mu=\nu=0$ now reads

$$
-\nabla^{2}\left[\frac{\epsilon}{2}\left(h_{00}-\varphi\right)\right]=-\kappa\left(T_{00}-\frac{1}{2} T\right) .
$$

Let us consider a configuration of matter distribution with low proper density $\rho$ moving at non-relativistic speed. According to the previous section, in an arbitrary Cartan gauge, 
the energy-momentum tensor of matter in this configuration will be given by (33), that is, $T_{\mu \nu}=\left(\rho c^{2}+p\right) e^{-2 \phi} g_{\mu \alpha} g_{\nu \gamma} u^{\alpha} u^{\gamma}-p e^{-\phi} g_{\mu \nu}$, where $\rho$ and $p$ are defined as invariant quantities with respect to Cartan transformations. Putting $e^{-\phi} \simeq 1-\epsilon \varphi$ and recalling that in a non-relativistic regime we can neglect $p$ with respect to $\rho$, we will have $T_{00}=$ $T \simeq \rho c^{2}$. In this way, we obtain, to first order in $\epsilon$, that $T_{\mu \nu} \simeq \rho c^{2} \eta_{\mu \alpha} \eta_{\nu \gamma} u^{\alpha} u^{\gamma}$. Finally, after substituting $\kappa=\frac{8 \pi G}{c^{4}}$ into (48), we obtain

$$
\nabla^{2}\left[\frac{\epsilon}{2}\left(h_{00}-\varphi\right)\right]=\frac{4 \pi G}{c^{2}} \rho
$$

which corresponds to the Poisson equation for the gravitational field $\nabla^{2} U=4 \pi G \rho$, with the gravitational potential $U$ given by (46).

\section{$5 \quad$ Different pictures of the same physical phenomena}

As we have shown in the previous section, when we go from one Cartan gauge $(M, g, \phi)$ to another $(M, \bar{g}, \bar{\phi})$ through a Cartan transformation (7), the gravitational field equations and the paths of motion of test particles and light rays do not change. It is important to note that the gravitational field in a Cartan gauge is described by the Riemann-Cartan curvature tensor, which is invariant and depends on two independent geometric elements: the metric and the torsion scalar field. Furthermore, due to Cartan transformations, the metric properties are decoupled from the parallel transport properties. Therefore, the same physical phenomena are described by distinct geometrical pictures, which arise in different Cartan gauges and require different physical interpretations related to either the metric or the torsion or both. Nonetheless, the numerical values of all physical quantities are the same in all gauges, since these quantities are invariant under Cartan transformations. This property rises the question about the existence of a gauge more suitable to perform the calculations of some physical quantity.

In fact, each gravitational field is described by a member of an equivalence class, the latter defined as the set of all Cartan gauges, with different metrics and different connections. In this class, one metric can be obtained from another and one connection can be obtained from another by means of a Cartan transformation. On the other hand, each equivalence class is completely determined by a solution of the Einstein equations in the Riemann gauge. The fact that the same gravitational field is described by an equivalence class of representations given by Cartan gauges may give rise to misinterpretations, since we are used to regard the gravitational field as a manifestation of the Riemannian curvature tensor, which depends only on the metric. This is particularly evident in the two special cases considered below.

The first case leads to an important equivalence class of Riemann-Cartan space-times. It is the class which characterizes the absence of gravitation, given by Minkowsky spacetime in the Riemann gauge $(M, \eta, 0)$, where both the curvature $\widehat{R}_{:: \beta \mu \nu}^{\alpha}[\eta, 0]=0$ and the

torsion $\widehat{T}_{:: \mu \nu}^{\alpha}[\eta, 0]=0$ vanish. Thus, in a Cartan gauge $\left(M, e^{\phi} \eta, \phi\right)$, the metric is given by $g_{\mu \nu}=e^{\phi} \eta_{\mu \nu}$ and the connection by $\Gamma_{: \mu \nu}^{\alpha}=\frac{1}{2} \phi_{, \mu} \delta_{: \nu}^{\alpha}$, where the torsion scalar field $\phi$ is the conformal factor of the Minkowski metric, according to the invariant relations (8)). These Riemann-Cartan space-times have two features, which are invariant under 
Cartan transformations. They satisfy the teleparallelism condition $R_{:: \beta \mu \nu}^{\alpha}\left[e^{\phi} \eta, \phi\right]=0$, and hence have a a Weitzenbi $i \frac{1}{2}$ ck connection [5]. They have a conformally flat metric, in the sense that $g_{\mu \nu}=e^{\phi} \eta_{\mu \nu}$, defined by the invariant condition $W_{:: \beta \mu \nu}^{\alpha}\left[e^{\phi} \eta, \phi\right]=0$ (as in general relativity). Therefore, the local causal structure is the same as the one of Minkowski space-time. Furthermore, test particles and light rays move on auto-parallel curves which are straight lines. In other words, in any Cartan gauge $\left(M, e^{\phi} \eta, \phi\right)$ there exists a "veiled" form of special relativity, the natural framework of which is, of course, Minkowski space-time in the Riemann gauge $(M, \eta, 0)$. Note that a Riemann-Cartan curvature $R_{:: \beta \mu \nu}^{\alpha}[g, \phi] \neq 0$, that is, a curved Riemann-Cartan space-time, is the necessary and sufficient condition for existence of a gravitational field.

The second case is given by the class of curved Riemann-Cartan space-times which are conformally flat in the Riemann gauge, i.e., where $\left(M, e^{-\phi} \eta, 0\right)$ and $R_{\beta \mu \nu}^{\alpha}\left[e^{-\phi} \eta, 0\right] \neq$ 0. In such situations, one can completely gauge away the Riemannian curvature by a Cartan transformation, thereby going to a Cartan gauge $(M, \eta, \phi)$ with Minkowski metric $\eta$, which determines the local causal structure, and a torsion scalar field $\phi$, which generates all dynamical properties of the gravitational field. This should not be regarded as being equivalent to the first case above, since the Riemann-Cartan curvature tensor $R_{\beta \mu \nu}^{\alpha}[\eta, \phi] \neq 0$ does not vanish. As in the previous case, light-rays move along null autoparallel curves that are straight lines, since the local causal structure is the same as that of Minkowski space-time. However, test particles move along time-like autoparallel curves that are not straight lines, considering that in this case the Riemann-Cartan spacetime is curved. This is well illustrated, for instance, when we consider in the Riemann gauge the class of Robertson-Walker (RW) metrics, which are known to be conformally flat [28]. If we go to the Cartan gauge $(M, \eta, \phi)$, by means of a Cartan transformation, we arrive at a new cosmological scenario in which a dynamical metric ceases to determine the cosmic expansion and other gravitational phenomena, all gravitational effects being now attributed to torsion through the sole action of a scalar field $\phi$ living in a curved Riemann-Cartan space-time with a non-dynamical Minkowski metric $\eta$.

There are many other examples of how distinct physical interpretations of the same phenomena are possible in different gauges. By way of illustration, we will consider, in this section, how one would describe, in a general Cartan gauge, an important effect predicted by general relativity: the so-called gravitational spectral shift. Let us consider the gravitational field generated by a massive body, which in an arbitrary Cartan gauge $(M, g, \phi)$ is described by both the metric tensor $g_{\mu \nu}$ and the torsion scalar field $\phi$. For the sake of simplicity, let us restrict ourselves to the case of a static field, in which neither $g_{\mu \nu}$ nor $\phi$ depends on time. Let us suppose that a light wave is emitted on the body at a fixed point with spatial coordinates $\left(r_{E}, \theta_{E}, \varphi_{E}\right)$ and received by an observer at the fixed point $\left(r_{R}, \theta_{R}, \varphi_{R}\right)$. Denoting the coordinate times of emission and reception by $t_{E}$ and $t_{R}$, respectively, the light signal, which in the Cartan gauge corresponds to a null affine geodesic, connects the event $\left(t_{E}, r_{E}, \theta_{E}, \varphi_{E}\right)$ with the event $\left(t_{R}, r_{R}, \theta_{R}, \varphi_{R}\right)$. Let $\lambda$ be an affine parameter along this null geodesic with $\lambda=\lambda_{E}$ at the event of emission and $\lambda=\lambda_{R}$ at the event of reception. If we write the line element in the form $d s^{2}=$ 
$g_{00}[r, \theta, \varphi] d t^{2}-g_{j k}[r, \theta, \varphi] d x^{j} d x^{k}$, then, since the geodesic is null, we must have

$$
g_{00}[r, \theta, \varphi]\left(\frac{d t}{d \lambda}\right)^{2}=g_{j k}[r, \theta, \varphi] \frac{d x^{j}}{d \lambda} \frac{d x^{k}}{d \lambda},
$$

so we can write

$$
\frac{d t}{d \lambda}=\left(\frac{g_{j k}[r, \theta, \varphi]}{g_{00}[r, \theta, \varphi]} \frac{d x^{j}}{d \lambda} \frac{d x^{k}}{d \lambda}\right)^{\frac{1}{2}} .
$$

On integrating between $\lambda=\lambda_{E}$ and $\lambda=\lambda_{R}$, we have

$$
t_{R}-t_{E}=\int\left(\frac{g_{j k}[r, \theta, \varphi]}{g_{00}[r, \theta, \varphi]} \frac{d x^{j}}{d \lambda} \frac{d x^{k}}{d \lambda}\right)^{\frac{1}{2}} d \lambda
$$

Because the integral on the right-hand side of the above equation depends only on the light path through space, and since the emitter and observer are at fixed positions in space, then $t_{R}-t_{E}$ has the same value for all signals sent. This implies that for any two signals emitted at coordinate times $t_{E}^{(1)}, t_{E}^{(2)}$ and received at $t_{R}^{(1)}, t_{R}^{(2)}$, we have $t_{R}^{(1)}-$ $t_{E}^{(1)}=t_{R}^{(2)}-t_{E}^{(2)}$, which means that the coordinate time difference $\Delta t_{E}=t_{E}^{(2)}-t_{E}^{(1)}$ at the event of emission is equal to the coordinate time difference $\Delta t_{R}=t_{R}^{(2)}-t_{R}^{(1)}$ at the event of reception. On the other hand, we know from Section 3 that the proper time recorded by clocks in a general Cartan gauge must be calculated by using the formula

$$
\Delta \tau=\int_{a}^{b}\left(e^{-\phi} g_{\mu \nu} \frac{d x^{\mu}}{d \lambda} \frac{d x^{\nu}}{d \lambda}\right)^{\frac{1}{2}} d \lambda .
$$

Therefore, the proper time recorded by the clocks of observers situated at the body and at the point of reception will be given, by the

$$
\Delta \tau_{E}=e^{-\frac{1}{2} \phi_{E}} \sqrt{g_{00}\left[r_{E}, \theta_{E}, \varphi_{E}\right]} \Delta t_{E},
$$

and

$$
\Delta \tau_{R}=e^{-\frac{1}{2} \phi_{R}} \sqrt{g_{00}\left[r, \theta_{R}, \varphi_{R}\right]} \Delta t_{R},
$$

where $\phi_{E}=\phi\left[r_{E}, \theta_{E}, \varphi_{E}\right]$ and $\phi_{R}=\phi\left[r_{R}, \theta_{R}, \varphi_{R}\right]$. Since $\Delta t_{E}=\Delta t_{R}$, we have the invariant relation

$$
\frac{\Delta \tau_{R}}{\Delta \tau_{E}}=\frac{e^{-\frac{1}{2} \phi_{R}} \sqrt{g_{00}\left[r_{R}, \theta_{R}, \varphi_{R}\right]}}{e^{-\frac{1}{2} \phi_{E}} \sqrt{g_{00}\left[r_{E}, \theta_{E}, \varphi_{E}\right]}} .
$$

Suppose now that $n$ waves of frequency $\nu_{E}$ are emitted in proper time $\Delta \tau_{E}$ from an atom situated on the body. Then $\nu_{E}=\frac{n}{\Delta \tau_{E}}$ is the proper frequency measured by an observer situated at the body. On the other hand, the observer situated at the fixed point $\left(r_{R}, \theta_{R}, \varphi_{R}\right)$ will see these $n$ waves in the proper time $\Delta \tau_{R}$, hence will measure a frequency $\nu_{R}=\frac{n}{\Delta \tau_{R}}$. Therefore, we have the invariant relation

$$
\frac{\nu_{R}}{\nu_{E}}=e^{\frac{1}{2}\left(\phi_{R}-\phi_{E}\right)} \frac{\sqrt{g_{00}\left[r_{E}, \theta_{E}, \varphi_{E}\right]}}{\sqrt{g_{00}\left[r_{R}, \theta_{R}, \varphi_{R}\right]}} .
$$


We, thus, see that $\nu_{R} \neq \nu_{E}$, i.e., the observed frequency differs from the frequency measured at the body, and this constitutes the spectral shift effect in a general Cartan gauge due to the gravitational field and, in general, depends on both $g_{00}$ and $\phi$. To conclude, two points related to the above equation are worth noting. The first is that, since in a Riemann gauge $\widehat{\phi}=0$, (52) reduces the well-known general relativistic formula for the gravitational spectral shift. The second point is that if we go to a Cartan gauge where $g_{00}$ is constant, then (152) becomes simply

$$
\frac{\nu_{R}}{\nu_{E}}=e^{\frac{1}{2}\left(\phi_{R}-\phi_{E}\right)}
$$

As we see, in this Cartan gauge all information concerning the gravitational spectral shift depends only on the torsion scalar field.

\section{Symmetries of space-time and scalar torsion}

In this section, we will consider the notion of space-time symmetry in the context of Riemann-Cartan space-times endowed with a scalar torsion field, which, in a certain sense, will generalize some known results of general relativity concerning isometries and Killing vector fields. We will also consider the conservation laws of auto-parallel motion in the Riemann-Cartan space-time.

Since in a Cartan gauge the gravitational field is described by two independent geometric objects, namely, the metric and the scalar torsion field, the definition of symmetry of the gravitational field should be given in terms of both. Also, this definition should be invariant under Cartan transformations and reduce to the usual definition in the Riemann gauge. Thus, taking into account the action of the Cartan transformations (77) on the fundamental elements $\left(g_{\mu \nu}, \phi\right)$ of the gravitational field, we define the symmetries of a Riemann-Cartan space-time with scalar torsion by the following conditions

$$
\mathfrak{L}_{k} g_{\mu \nu}=f g_{\mu \nu}, \mathfrak{L}_{k} \phi=f
$$

where $f$ is an arbitrary function and $\mathfrak{L}_{k}$ is the Lie derivative with respect to the vector field $k^{\mu}=\frac{d x^{\mu}}{d \lambda}$, referred to as a Killing-Cartan vector field, since it behaves as a Killing vector field in the Riemann gauge. However, considering that in the equations (53) above the Lie derivative of the torsion scalar field $\mathfrak{L}_{k} \phi=k[\phi]=\phi_{, \mu} k^{\mu}=\frac{d \phi}{d \lambda}$ is equal to the conformal factor $f$ of the metric, the symmetries of a Riemann-Cartan space-time with scalar torsion can alternatively be defined by the equations

$$
\mathfrak{L}_{k} g_{\mu \nu}=g_{\mu \nu} k[\phi]=g_{\mu \nu} \frac{d \phi}{d \lambda},
$$

which makes no reference to the arbitrary function $f$ and has the advantage of being invariant under Cartan transformations. As we have seen, assuming the invariance of the contravariant components of the Killing-Cartan vector field under Cartan transformations $\widehat{k}^{\mu}=k^{\mu}=\frac{d x^{\mu}}{d \lambda}$ also assures the invariance of the equations (54) under Cartan transformations (17). Moreover, the definition given by (54) reduces to the isometry definition in the Riemann gauge, in accordance with the symmetry definition of the gravitational field in 
general relativity. Note that the definition (54) of symmetries of Riemann-Cartan spacetimes can also be obtained from the definition $\mathfrak{L}_{k} \eta_{\mu \nu}=0$ of symmetries of Minkowski space-time in special relativity, through the coupling prescription $\eta_{\mu \nu} \rightarrow e^{-\phi} g_{\mu \nu}$.

The generalization of the Killing equations in a Cartan gauge can be obtained from (54) as follows. Substituting the Lie derivative of the metric, expressed in terms of the Riemann-Cartan covariant derivative, given by $\mathfrak{L}_{k} g_{\mu \nu}=\widetilde{\nabla}_{\nu} k_{\mu}+\widetilde{\nabla}_{\mu} k_{\nu}=k^{\alpha} \nabla_{\alpha} g_{\mu \nu}+$ $\nabla_{\nu} k_{\mu}+\nabla_{\mu} k_{\nu}-\frac{1}{2}\left(\phi_{, \mu} k_{\nu}+\phi_{, \nu} k_{\mu}\right)+k^{\alpha} \phi_{, \alpha} g_{\mu \nu}$ in (54), and taking into account the metricity condition $\nabla_{\alpha} g_{\mu \nu}=0$, we obtain that the Killing-Cartan vector fields must be solution of the equations

$$
\nabla_{\mu} k_{\nu}+\nabla_{\nu} k_{\nu}-\frac{1}{2}\left(k_{\mu} \phi_{, \nu}+k_{\nu} \phi_{, \mu}\right)=\widetilde{\nabla}_{\nu} k_{\mu}+\widetilde{\nabla}_{\mu} k_{\nu}-k^{\alpha} \phi_{, \alpha} g_{\mu \nu}=0
$$

which will be referred to as Killing-Cartan equations. It is easily seen that they coincide with the Killing equations in the Riemann gauge where $\phi=0$. Therefore, the symmetries of the gravitational field in a Cartan gauge are given by the Killing-Cartan vector fields, which depend on both the metric and the torsion scalar field. Clearly, the properties of the gravitational field do not change along the integral curves of these vector fields.

Note that the Killing-Cartan equations (55) can also be obtained from the Killing equations in Minkowski space-time $k_{\nu, \mu}+k_{\mu, \nu}=0$, given with respect to a Lorentz coordinate system in special relativity. Let us consider, by definition, that the contravariant components of the Killing vector field are invariant under Cartan transformations $\widehat{k}^{\mu}=k^{\mu}$. Then, from $\widehat{k}_{\nu}=\widehat{g}_{\nu \mu} \widehat{k}^{\mu}=e^{-\phi} g_{\nu \mu} k^{\mu}=e^{-\phi} k_{\nu}$ and applying the coupling prescription (28) to the Killing equations $k_{\nu, \mu}+k_{\mu, \nu}=0$, we obtain that $\widehat{\nabla}_{\mu} \widehat{k}_{\nu}+\widehat{\nabla}_{\nu} \widehat{k}_{\mu}=$ $e^{-\phi}\left[\nabla_{\mu} k_{\nu}+\nabla_{\nu} k_{\nu}-\frac{1}{2}\left(k_{\mu} \phi_{, \nu}+k_{\nu} \phi_{, \mu}\right)\right]=0$, which are equivalent to the Killing-Cartan equations (55).

It should be noted that the Killing-Cartan vector fields also coincide with the Killing vector fields in a Cartan gauge where $\mathfrak{L}_{k} \phi=k[\phi]=\frac{d \phi}{d \lambda}=0$, that is, when the torsion scalar field is invariant along the integral curve of the Killing-Cartan vector field $k^{\mu}$, since the Killing-Cartan equations (54) coincide with the Killing equations.

To conclude this section let us note that the conservation laws of motions along autoparallel curves are closely related to the existence of Killing-Cartan vector fields, and are very useful to solve the equations of motion of test particles and light rays in a Cartan gauge. Thus, consider a test particle whose path is an auto-parallel time-like curve $x^{\mu}(\tau)$, parametrized with proper time $\tau$ and 4 -velocity vector $u^{\mu}=\frac{d x^{\mu}}{d \tau}$. Then, the invariant quantity

$$
C=e^{-\phi} g_{\mu \nu} k^{\mu} u^{\nu}
$$

defined in an arbitrary Cartan gauge, where $k^{\mu}$ is a Killing-Cartan vector field, is not only an invariant under Cartan transformations, but also a constant of motion. Considering that

$$
\frac{d C}{d \lambda}=u^{\alpha} \nabla_{\alpha} C=e^{-\phi}\left[g_{\alpha \beta} k^{\alpha} u^{\mu} \nabla_{\mu} u^{\beta}-\frac{d \phi}{d \tau} g_{\alpha \beta} k^{\alpha} u^{\beta}+\frac{1}{2}\left(\nabla_{\mu} k_{\nu}+\nabla_{\nu} k_{\nu}\right) u^{\mu} u^{\nu}\right]
$$

and substituting $\left(\nabla_{\mu} k_{\nu}+\nabla_{\nu} k_{\nu}\right) u^{\mu} u^{\nu}=\frac{d \phi}{d \tau} g_{\alpha \beta} k^{\alpha} u^{\beta}$ which was obtained from the KillingCartan equations (155), we obtain that 


$$
\frac{d C}{d \lambda}=u^{\alpha} \nabla_{\alpha} C=e^{-\phi} g_{\alpha \beta} k^{\alpha}\left(u^{\mu} \nabla_{\mu} u^{\beta}-\frac{d \phi}{d \tau} u^{\beta}\right)=0,
$$

taking into account the equations of motion of the test particle given by (25).

As an example, let us consider, in a Cartan gauge, the gravitational field equations (47) with $T_{\mu \nu}=\Lambda=0$, which is given by $R_{\mu \nu}[g, \phi]=0$. Clearly, a solution of the above equations is given by the metric

$$
d s^{2}=c^{2} d t^{2}-\frac{1}{\left(1-\frac{2 m}{r}\right)^{2}} d r^{2}-\frac{r^{2}}{1-\frac{2 m}{r}}\left(d \theta^{2}+\operatorname{sen}^{2} \theta d \psi^{2}\right)
$$

and the torsion scalar field

$$
\phi(r)=-\ln \left(1-\frac{2 m}{r}\right) .
$$

Note that the solution above is asymptotically flat, since $\left(1-\frac{2 m}{r}\right) \rightarrow 1$ and $\phi(r) \rightarrow 0$ when $r \rightarrow \infty$, while the constant $m$ can be identified as the mass of the spherical matter distribution. In the weak field limit $(r \gg 2 m)$, we obtain that the Newtonian potential (46) of the above solution is given by $U=-\frac{c^{2} m}{r}$. Since the Newtonian potential of a spherical distribution of mass with mass $M$ at a distance $r$ from its center is $U=-\frac{G M}{r}$, where $G$ is the gravitational constant, it follows that $m=\frac{G M}{c^{2}}$. Surely, this solution is a member of the equivalence class which corresponds to Schwarschild solution of general relativity. One can easily verify that the solution (59) and (60) can be transformed, through a Cartan transformation (77) with conformal factor $f(r)=\ln \left(1-\frac{2 m}{r}\right)$, into the representation of the gravitational field in the Riemann gauge which is given by Schwarzschild solution.

This gravitational field has two Killing-Cartan vector fields given by the time-like vector field $k^{\mu}=\delta_{0}^{\mu}$ and the space-like vector field $w^{\mu}=\delta_{3}^{\mu}$, since both $g_{\mu \nu}$ and $\phi$ do not depend on the coordinates $x^{0}=c t$ and $x^{3}=\psi$. Note that they are also Killing vector fields, as $k^{\mu} \phi_{, \mu}=0$ and $w^{\mu} \phi_{, \mu}=0$. Therefore, according to our previous results, a test particle moving in this gravitational field, along a time-like auto-parallel curve $x^{\mu}(\tau)$ with 4-velocity $u^{\mu}=\frac{d x^{\mu}}{d \tau}$, has the following constants of motion $E=e^{-\phi} g_{\alpha \beta} k^{\alpha} u^{\beta}=\left(1-\frac{2 m}{r}\right) u^{0}$ and $L=e^{-\phi} g_{\alpha \beta} w^{\alpha} u^{\beta}=-r^{2} \sin ^{2} \theta u^{3}$ given, respectively, by the energy per unit mass and angular momentum per unit mass.

The constants of motion $E$ and $L$ above can be used to obtain the equation of the orbit of a test particle in this gravitational field as follows. Let us assume, for simplicity, that the particle is moving in the plane $\theta=\frac{1}{2} \pi$. In a Cartan gauge the norm of the 4 -velocity $u^{\mu}=\frac{d x^{\mu}}{d \tau}$ of the test particle is such that $g_{\mu \nu} u^{\mu} u^{\nu}=e^{\phi}$. Thus, using the expressions for $E$ and $L$ above to substitute the components $u^{0}=\frac{d x^{0}}{d \tau}=\frac{E}{\left(1-\frac{2 m}{r}\right)}$ and $u^{3}=\frac{d \psi}{d \tau}=-\frac{L}{r^{2}}$ into $g_{\mu \nu} u^{\mu} u^{\nu}=e^{\phi}$, it follows that $E^{2}-\left(u^{2}\right)^{2}-\left(\frac{L^{2}}{r^{2}}+1\right)\left(1-\frac{2 m}{r}\right)=0$. Now, substituting $u^{2}=\frac{d r}{d \psi} \frac{d \psi}{d r}=-\frac{L}{r^{2}} \frac{d r}{d \psi}$, we obtain that

$$
E^{2}-\frac{L^{2}}{r^{4}}\left(\frac{d r}{d \psi}\right)^{2}-\left(\frac{L^{2}}{r^{2}}+1\right)\left(1-\frac{2 m}{r}\right)=0 .
$$

Finally, integrating the above expression (61) we obtain the equation of the orbit $r=r(\psi)$, which is identical to the equation of the orbit in Schwarzschild space-time. Similar results hold with respect to the motion of light rays. Therefore, the static and spherical symmetric 
gravitational field, represented in a Cartan gauge by (159) and (60), is in accordance with all classical test of general relativity.

\section{Final remarks}

In this work, we present a scenario in which the gravitational field is not associated only with the metric tensor, but with the combination of both the metric $g_{\mu \nu}$ and a geometrical scalar field $\phi$. This formulation led to a new kind of invariance, which involves simultaneous transformation of $g_{\mu \nu}$ and $\phi$. Moreover, we have shown that in geometrical setting the same physical phenomena may appear in different pictures and distinct representations. For instance, in the case of the gravitational spectral shift, except in the Riemann gauge, in all other representations the torsion scalar field plays an essential role. An important conclusion to be drawn from what has been presented in this paper is that general relativity can be recast in a Riemann-Cartan space-time. In close connection with this, it should be mentioned that by using a rather similar procedure it has been shown recently that general relativity can also be formulated in another non-Riemannian setting, namely, that of Weyl integrable space-time [34]. The possibilities of enlarging the set of mathematical frameworks that are capable of describing the same physical theory, in this case, general relativity, using distinct geometrical languages, seems to give an illustration of the epistemological view conceived by $\mathrm{H}$. Poincaré that the geometry of space-time is perhaps a convention that can be freely chosen by the theoretician [35, 36].

\section{Acknowledgments}

Carlos Romero and Silvina Paola Gomez Martinez would like to thank CNPq and CAPES for financial support.

\section{References}

[1] F.W. Hehl, J.D. McCrea and E.W. Mielke and Y. Ne'eman, Phys. Rep. 258, 1 (1995), arxiv:gr-gc/9402012.

[2] J.A. Schouten, Ricci-Calculus ( Second Edition, Springer 1954).

[3] H. Goenner, Living Rev. Rel. 7, 2 (2004).

[4] E. Cartan, Comptes Rendus Acad. Sci (Paris) 174, 437 (1922). For a review with extensive bibliography see F. W. Hehl, P. von der Heyde, G. D. Kerlick, J. M. Nester, Rev. Mod. Phys. 48, 393 (1976). See also M. Gasperini and V. De Sabatta, Introduction to Gravitation (World Scientific, 1986). V. de Sabatta and C. Sivaram, Spin and Torsion in Gravitation (World Scientific, 1994). R. Kerner, Ann. Inst. H. Poincaré, 34, 473 (1981). A. Trautman, Einstein-Cartan theory in Encyclopedia of Mathematical Physics, vol. 2, pages 189-195, Ed. J. P. Françoise, G. L. Naber and Tsou S.T. (Oxford: Elsevier, 2006). Y. Mao, M. Tegmark, A. H. Guth, and S. Cabi, Phys. Rev. D76, 104029 (2007), arXiv:gr-qc/0608121. 
[5] For a review on other gravitational theories making use of the concept of torsion see R. Aldrovandi and J. G. Pereira, e-Print arXiv:0801.4148v1. R. Utiyama, Phys. Rev. 101, 1597 (1956); T.W.B. Kibble, J. Math. Phys. 2, 212 (1961); F. W. Hehl, P. Von Der Heyde, G. D. Kerlick, and J. M. Nester. Rev. Mod. Phys., 4, 393 (1976); A. Trautman, Symp. Math. 12, 139 (1973); A. Trautman, arXiv:gr-qc/0606062. F.W. Hehl, Four lectures on Poincare gauge field theory, in Proceedings of the 6th School of Cosmology and Gravitation, Erice, Italy, (1979). Y.N. Obukhov, Poincaré gauge gravity: Selected topics, Int. J. Geom. Meth. Mod. Phys. 3 (2006) 95-138, gr-qc/0601090. G. R. Bengochea and R. Ferraro, Phys. Rev. D 79, 124019 (2009). E. V. Linder, Phys. Rev. D 81, 127301 (2010).

[6] For a review, see T. P. Sotiriou and V. Faraoni, Rev. Mod. Phys. 82, 451 (2010) and the references therein.

[7] S. Capozziello, M. De Laurentis, Phys. Rep. 509, 167 (2011), arXiv:1108.6266.

[8] S. L. Adler, Rev. Mod. Phys. 54, 729 (1982). P. D. Mannheim, Found. Phys. 42, 338 (2012). P. D. Mannheim, Gen. Rel. Grav. 22, 289 (1990).

[9] H. Weyl, Sitzungesber Deutsch. Akad. Wiss. Berli 465 (1918); H. Weyl, Space, Time, Matter (Dover, New York, 1952).

[10] For a nice review on Weyl geometry see E. Scholz, arXiv:1111.3220.

[11] M. Novello and H. Heintzmann, Phys. Lett. A 98, 10 (1983); K. A. Bronnikov, Yu. M. Konstantinov and V. N. Melnikov, Grav. Cosmol. 1, 60 (1995); M. Novello, L.A.R. Oliveira, J.M. Salim and E. Elbas, Int. J. Mod. Phys. D 1, 641 (1993); J. M. Salim and S. L. Sautú, Class. Quant. Grav 13, 353 (1996); H. P. de Oliveira, J. M. Salim and S. L. Sautú, Class. Quant. Grav. 14, 2833 (1997); V. Melnikov, Classical Solutions in Multidimensional Cosmology in Proceedings of the VIII Brazilian School of Cosmology and Gravitation II, ed. M. Novello (Editions Frontières, 1995) p.542; R. G. Gannouji, H. Nandan, N. Dadhich, JCAP 11, 51 (2011). O. Arias, R. Cardenas and I.Quiros, Nucl. Phys. B 643, 187 (2002); J. Miritzis, Class. Quant .Grav. 21, 3043 (2004); J. Miritzis, J.Phys.: Conf. Ser. 8, 131 (2005); M. Israelit, Found. Phys. 35, 1725 (2005); F. Dahia, G. A. T. Gomez and C. Romero, J. Math.Phys. 49, 102501 (2008); J. E. Madriz Aguilar and C. Romero, Found. Phys. 39, 1205 (2009). T. Moon, J. Lee, P. Oh, Mod. Phys. Lett. A 25, 3129 (2010), arXiv.gr-qc/0912.0432.

[12] G. Germàn, Phys. Rev. D 32, 3307(1985). I. L. Shapiro, Phys. Rept.357:113, 2002 and the references therein.

[13] A. Einstein, The Meaning of Relativity, Princeton University Press (1970).

[14] S. Capozziello, G. Lambiase and C. Stornaiolo, Geometric classification of the torsion tensor of space-time, arXiv:gr-qc/0101038.

[15] For a review see Richard T Hammond Rep. Prog. Phys. 65 (2002) 599; Yongsung Yoon, Phys.Rev. D59 (1999) 127501, arXiv:9904018v1; C. J. Park, Yongsung Yoon, 
Gen.Rel.Grav. 29 (1997) 765, arXiv: 9611053v1; Sung-Won Kim Phys. Rev. D 34 (1986), 1011; V. De Sabbata and M. Gasperini Phys. Rev. D 23(1981), 2116; Wei-Tou Ni Phys. Rev. D 19 (1979), 2260; S. Hojman, M. Rosenbaum, M. P. Ryan, and L. C. Shepley Phys. Rev. D 17 (1978), 3141;

[16] Robert M. Wald, General Relativity, The University of Chicago Press (1984).

[17] J. F. T. Giglio and W. A. Rodrigues Jr., Ann. Phys, 524, 302 (2012). ArXiv: 1111.2206.

[18] S. R. Mainwaring and G. E. Stedman, Phys. Rev. A 47, 3611 (1993).

[19] F. P. Poulis and J. M. Salim, Int. J. Mod. Phys.: Conf. Series, 3, 87 (2011), arXiv:grqc/1106.3031.

[20] C. H. Brans and R. H. Dicke, Phys. Rev. 124, 925 (1961). R. H. Dicke, Phys. Rev. 125, 2163 (1962).

[21] R. Adler, M. Bazin and M. Schiffer, Introduction to General Relativity (McGraw-Hill, 1975).

[22] Canuto, P. J. Adams, S. H. Hsieh, E. Tsiang, Phys. Rev. D 16, 1643 (1977).

[23] M. P. Dabrowski, T. Denkiewicz and D. Blaschke, Annalen Phys. 16, 237 (2007)

[24] V. Faraoni, Cosmology in Scalar-Tensor Gravity (Kluwer Academic Publishers, Dordrecht, 2004).

[25] P. A. M. Dirac, Nature, 139, 323 (1937).

[26] R. T. Singh and D. Shridhar, Int. J. Theor. Phys. 26, 901 (1987).

[27] C. Romero, J. B. Fonseca-Neto and M. L. Pucheu, Found. Phys. 42, 224 (2012), arXiv: gr-qc/1101.5333.

[28] M. Ibison, J. Math. Phys 48, 122501 (2007).

[29] V. Faraoni, Int. J. Theor. Phys, 38, 217 (1999). V. Faraoni, E. Gunzig and P. Nardoni, Fund. Cosm. Phys. 20, 121 (1999). V. Faraoni and S. Nadeau, Phys. Rev. D 75, 23501 (2007).

[30] I. Quiros, R. Bonal and R. Cardenas, Phys. Rev. D 62, 044042 (2000). I. Quiros, R. Garxía-Salcedo and J. E. Madriz Aguilar, arXiv: gr-qc/1108.2911v2. I. Quiros, R. Garxía-Salcedo, J. E. Madriz Aguilar and T. Matos, arXiv: gr-qc/1108.5857v2.

[31] S.W. Hawking and G.F.R. Ellis, The Large scale structure of space-time, Cambridge University Press, Cam- bridge (1973).

[32] See, for instance, M. Novello, "Theoretical Cosmology", in VII Brazilian School of Cosmology and Gravitation, Ed. M. Novello (Editions Frontières, 1995). Iain A. Brown and A. Hammami, arXiv: gr-qc/1112.0575v2 (2011). 
[33] M. P. Dabrowski, T. Denkiewicz and D. Blaschke, Annalen Phys. 16, 237 (2007).

[34] C. Romero, J. B. Fonseca-Neto and M. L. Pucheu, Class. Quantum Grav. 29, 155015 (2012). arXiv:1201.1469.

[35] H. Poincaré, Science and Hypothesis (Dover, New York, 1952).

[36] I. W. Roxburgh and R. K. Tavakol, Found. Phys. 8, 229 (1978).

[37] H. Stephani, D. Kramer, M.A.H. MacCallum, C. Hoenselaers and E. Herlt, Exact solutions of Einstein's field equations, Cambridge Univ. Press, UK (2003).

[38] S. Kobayashi and K. Nomizu, Foundations of Differential Geometry, Vol. I, (Interscience Publishers, N.Y., London,1963). 